\title{
SOX2-phosphorylation toggles a bistable differentiation-switch in squamous cell carcinoma
}

\author{
Steven Hoang-Phou ${ }^{1}$, Ana Sastre-Perona ${ }^{1,5}$, Matteo Abbruzzese ${ }^{1}$, Zhe Ying ${ }^{4}$, Jasmin Siegle ${ }^{1}$, \\ Beatriz Aranda Orgilles ${ }^{3}$, Pedro P. Rocha ${ }^{3,6}$, Iannis Aifantis ${ }^{3}$, Jane Skok ${ }^{3}$, Slobodan Beronja ${ }^{4}$, \\ and Markus Schober ${ }^{1,2,3,{ }^{*}}$
}

1. The Ronald O. Perelman Department of Dermatology, New York University Grossman School of Medicine, New York, NY, USA

2. Department of Cell Biology, New York University Grossman School of Medicine, New York, NY, USA

3. Department of Pathology,New York University Grossman School of Medicine, New York, NY, USA

4. Division of Human Biology, Fred Hutchinson Cancer Research Center, Seattle, WA, USA

5. Experimental therapies and Novel Biomarkers in Cancer, Hospital La Paz Institute fro Health Research (IdiPAZ), Madrid, Spain

6. National Institute of Child Health and Human Development, National Institutes of Health, Bethesda, MD, USA

* Corresponding Author and Lead Contact

The Ronald O. Perelman Department of Dermatology

New York University School of Medicine

522 First Avenue, Smilow Research Building. 411

New York, NY-10016

Markus.Schober@nyulangone.org

Tel \# 212-263-9251

Fax \# 212-263-581 


\section{Summary}

The fate choice between stem cell self-renewal and differentiation is regulated by bistable transcriptional networks, which are balanced in homeostasis and imbalanced in tumors. Yet, how stem cells switch from self-renewal to differentiation remains a conundrum. Here, we discover a molecular mechanism that allows stem cell-like tumor propagating cells (TPCs) in squamous cell carcinomas (SCCs) to switch from a mutually exclusive SOX2PITX1-TP63 self-renewal circuit to a KLF4 driven differentiation program, dependent on the relative occupancy of a novel KIf4-regulatory enhancer cluster $\left(K I f 4^{E C 944}\right)$ by SOX2 or KLF4, respectively. We find SOX2 occupies this site in TPCs to inhibit KIf4 transcription, but upon phosphorylation SOX2 becomes evicted from KIf4 ${ }^{E C 944}$, allowing residual KLF4 to occupy this site instead, boost the expression of KLF4 and its downstream targets, and differentiate self-renewing TPCs into post-mitotic SCC cells. This mechanism allows SOX2 to promote self-renewal and tumor formation, while preserving the differentiation potential in SCC cells. Our data suggest that stochastic cell fate decisions depend on the effective concentration of enzymatically regulated transcription factors. The surprising specificity by which SOX2-phosphorylation governs the bistable KIf4 ${ }^{E C 944}$ network-switch in SCCs reveals a conceptual framework for the identification of similar switches in other stem cell and cancer types and their potential development into cell type specific differentiation therapies for diseases in which tissue homeostasis has gone awry. 


\section{Introduction}

The binary fate choice between stem cell self-renewal or differentiation defines the rate of clonal expansion within tissues (Blanpain and Simons, 2013; Simons and Clevers, 2011). Although self-renewal and differentiation are neutrally balanced in tissue homeostasis (Klein and Simons, 2011), they become skewed towards self-renewal in cancer (Driessens et al., 2012; Klein et al., 2009). It has therefore been speculated that increased self-renewal and aberrant differentiation are the root cause of cancerous growth and the molecules controlling stemness could present effective therapeutic targets (Kreso and Dick, 2014).

Mounting evidence from genetic studies suggest that binary cell fate changes are governed by bi-stable transcriptional networks (Davis and Rebay, 2017; Young, 2011). These bi-stable networks allow cells to assume one of two mutually exclusive stable cell states, where each state is determined by master regulatory transcription factors. To maintain a specific cell state, master regulatory transcription factors co-operatively enhance each other's expression in a positive feedback circuit, while they inhibit transcriptional programs that specify their opposing cell fate. Although cell fate defining master regulatory transcription factors and their transcriptional targets have been identified in several homeostatic tissues and cancers, it is still unclear how stem cells switch from self-renewal to differentiation.

Stratified epithelia and SCCs emerged as powerful model systems to address this longstanding question on a molecular level. Cutaneous SCCs are - similar to normal epidermis hierarchically organized and maintained by stem cell-like cells that are located along the tumorstroma interface (Pierce and Wallace, 1971; Schober and Fuchs, 2011). TPCs proliferate to selfrenew or they differentiate into post-mitotic SCC cells without tumorigenic potential. Quantitative lineage tracing supports the idea that stem cell-like cells maintain SCC growth and their cell fate outcomes are stochastically determined when cells divide, with a bias towards self-renewal to allow for the geometric tissue expansion as squamous differentiation becomes increasingly aberrant as tumors progress (Driessens et al., 2012).

The transcription factor SOX2 is responsible for clonal expansion and SCC growth (Boumahdi et al., 2014; Siegle et al., 2014). Although Sox2 is epigenetically repressed and not detected in normal skin epithelial cells (Arnold et al., 2011; Ezhkova et al., 2011), it becomes expressed de novo in TPCs (Schober and Fuchs, 2011; Siegle et al., 2014). Once SOX2 is expressed, it is specifically detected in nuclei of TPCs, but not differentiated SCC cells (Boumahdi et al., 2014; Siegle et al., 2014). SOX2 co-localizes and physically interacts with the transcription factors 
$\Delta$ Np63 (Jiang et al., 2020; Watanabe et al., 2014) and PITX1 (Sastre-Perona et al., 2019), and these transcription factors co-operatively promote each other's transcription in a positive feedback circuit that drives TPC self-renewal, as they inhibit Klf4 expression and squamous differentiation.

The transcription factor KLF4 is expressed in supra-basal epidermal keratinocytes where it promotes the differentiation of stem cell-like (basal) into post-mitotic (supra-basal) squamous epithelial cells, cornification and epidermal barrier formation (Fuchs et al., 1999). Although the regulation and function of Klf4 in epidermal differentiation is still unclear and context dependent (Sen et al., 2012; Szigety et al., 2020), genetic gain- and loss-of-function studies suggest that KLF4 restricts SOX2, PITX1, and $\triangle$ Np63 expression and proliferation to the basal SCC layer (Sastre-Perona et al., 2019), consistent with its tumor suppressive functions (Li et al., 2012).

These gain- and loss-of-function data suggest that TPC self-renewal and differentiation are regulated by a bi-stable transcriptional network of mutually exclusive SOX2 -PITX1- $\triangle$ Np63 and KLF4 dependent gene expression programs, but it remained a conundrum how some TPCs can exit their self-renewal circuit in steady-state tumors and differentiate, when the expression of KLF4, which needs to inhibit the self-renewal circuit, is still repressed by the self-renewal program.

Here, we identify the molecular mechanism that solves this self-renewal and differentiation paradox. We find that SOX2 binds to Klf4 enhancer cluster EC944 to inhibit Klf4 transcription and this promotes TPC self-renewal and SCC growth. However, SOX2 is specifically evicted from EC944 when SOX2 becomes phosphorylated on Serine S39 and S253 in mouse and corresponding S37 and S251 in human SCCs allowing residual KLF4 to bind KIf4 ${ }^{E C 944}$ instead of SOX2. KLF4 binding initiates an auto-regulatory transcriptional program promoting KLF4 expression along with other squamous differentiation markers, as increased occupancy of KLF4 at gene regulatory enhancers decommission the TPC self-renewal program. Conceptually, these data suggest that the mutually exclusive occupancy of a single transcriptional enhancer by two opposing master-regulatory transcription factors defines the binary fate choice between stem cell self-renewal and differentiation, and their occupancy of this enhancer is governed by enzymatically regulated, post-translational transcription factor modifications. The simplicity and specificity of this enzymatically regulated bi-stable switch between two stable cell states is appealing because it informs the development of differentiation therapies for cancers and other diseases where stem cell self-renewal and differentiation have gone awry.

\section{Results}

SOX2 is phosphorylated on Serine-37 and Serine-251 in human squamous cell carcinoma 
Because we detected multiple SOX2 bands in SCC lysates (Siegle et al., 2014) and transcription factor activities can change rapidly upon phosphorylation (Whitmarsh and Davis, 2000), we wondered whether SOX2 was phosphorylated in SCCs and whether SOX2phosphorylation could influence TPC self-renewal. To test this hypothesis, we extracted total SCC protein lysates and separated them along with calf intestinal phosphatase (CIP) treated control lysates on PhosTag gels (Figure 1A). The Phos-Tag reagent binds to phosphorylated amino acids and it reduces the mobility of phosphorylated proteins when added to poly-acrylamide gels (Kinoshita et al., 2006). Western blotting revealed four SOX2 bands and the two largest bands vanished upon CIP treatment, indicating that a fraction of SOX2 is phosphorylated in SCCs.

To determine which SOX2 amino acids are phosphorylated in SCCs, we first fused two FLAG and two Streptavidin tags to the N-terminal end of human SOX2 (F-SOX2) (Figure 1B). Human and mouse SOX2 are almost identical in their amino acid sequences and they differ only by an insertion of two glycine residues at position 23-24 in mouse SOX2. We expressed our F-SOX2 transgenes in human SCC cultures close to endogenous levels, purified large amounts of F-SOX2 by immuno-precipitation, digested it with cyanogen bromide-Trypsin, and identified phosphorylated peptides with tandem mass-spectrometry (Figure S1A-C). This approach identified two phosphorylated peptides with fragmentation patterns corresponding to human SOX2 that is phosphorylated on Serine 37 (S37) and Serine 251 (S251) (Figure 1B, S1D-E). Confocal microscopy with site specific phospho-SOX2 antibodies detected pS253-SOX2 in mouse (Figure 1C) and corresponding pS251-SOX2 in human (Figure S1F) SCC sections, indicating that endogenously expressed SOX2 is phosphorylated in primary SCCs.

To functionally test if SOX2 S37 and S251 phosphorylation affects SOX2 activity, we mutated both serine residues in our F-SOX2 transgene to either alanine (A) or aspartic acid (D) before we expressed them in SCC cells. Consistent with the notion that alanine cannot be phosphorylated and aspartic acid is similar to phosphorylated serine in charge and structure, we detected endogenously expressed SOX2 with anti-pS251-SOX2 antibodies along with the larger F-SOX2 ${ }^{\mathrm{WT}}$ and F-SOX2 ${ }^{\mathrm{DD}}$, but not the F-SOX2 $2^{\mathrm{AA}}$ transgene by western blotting (Fig 1D). In addition, antiFLAG antibodies detected only a single F-SOX2 ${ }^{\mathrm{AA}}$ band on Phos-Tag gels, whereas F-SOX2 ${ }^{\mathrm{WT}}$ showed a multi-band pattern (Figure S1G), mirroring that of endogenously expressed SOX2 (Figure1A). Together, these data show that endogenous SOX2 and F-SOX2 transgenes are phosphorylated on S37 and S251 in SCCs and these two sites are either the only phosphorylated SOX2 amino acids or their phosphorylation is required for additional phosphorylation events in SCC cells. 


\section{F-SOX2 $2^{\mathrm{AA}}$ clones outcompete F-SOX2 ${ }^{\mathrm{DD}}$ expressing clones in squamous cell carcinomas.}

To test if SOX2-phosphorylation affects its activity, we turned to a clonal competition model (Ge et al., 2017; Siegle et al., 2014), which allows us to directly compare the relative expansion of $F-S O X 2^{D D} ; H 2 B-R F P$ and $F-S O X 2^{A A} ; H 2 B-G F P$ expressing cells within the same tumor over time. We generated lentiviruses for each transgene and combined them at equivalent titers before infecting primary TPC cultures at low multiplicity of infection. FACS analyses confirmed $\sim 5 \%$ of TPCs expressed either $F-S O X 2^{D D} ; H 2 B-R F P$ or $F-S O X 2^{A A} ; H 2 B-G F P$ before we transplanted them orthotopically into mouse dermis (Figure S1H). Two weeks after transplantation, we measured how $F-S O X 2^{A A} ; H 2 B-G F P$ cells expanded in comparison to $F-S O X 2^{D D} ; H 2 B-R F P$ cells within the same tumor (Figure 1E). FACS analyses revealed that SOX2 ${ }^{\mathrm{AA}}$ expressing clones expand faster than SOX $2^{\mathrm{DD}}$ expressing clones in both, mouse and human SCC transplantation models (Figure 1F-G, S1H). Likewise, confocal microscopy also detected more $F$-SOX $2^{A A} ; H 2 B-G F P$ compared to $F-S O X 2^{D D} ; H 2 B-R F P$ expressing cells in SCC sections (Figure $1 \mathrm{H}$ ). Interestingly, $F$ $S O X 2^{A A} ; H 2 B-G F P$ expressing cells were frequently detected in the basal SCC layer along the tumor-stroma interface, whereas $F-S O X 2^{D D} ; H 2 B-R F P$ expressing cells were more commonly detected in differentiated, post-mitotic tumor parts. This differential localization pattern suggested that SOX2 phosphorylation inhibits TPC self-renewal or enables the differentiation of TPCs into post-mitotic SCC cells even when endogenous SOX2 is also expressed in these SCC cells.

\section{Ectopic SOX2 expression accelerates squamous cell carcinoma initiation in a phosphorylation dependent manner.}

Because Sox2 is epigenetically repressed and not detected in normal skin epithelial cells (Arnold et al., 2011; Ezhkova et al., 2011), but becomes de novo expressed in TPCs of cutaneous SCCs (Boumahdi et al., 2014; Schober and Fuchs, 2011; Siegle et al., 2014), we wondered whether ectopic SOX2 expression in skin epithelial cells would promote SCC initiation and growth and whether tumor initiation rates would vary with SOX $2^{\mathrm{WT}}, \mathrm{SOX} 2^{\mathrm{AA}}$ and SOX2 ${ }^{\mathrm{DD}}$ expression. To address these questions, we transduced Hras $^{\mathrm{G12V}-K I} ; R 26-L S L-Y F P$ mice at e9.5 (Beronja et al., 2010) with lentiviruses expressing either CRE alone (red), or CRE along with SOX2 ${ }^{\mathrm{WT}}$ (brown), $\mathrm{SOX}^{\mathrm{AA}}$ (blue), or SOX2 ${ }^{\mathrm{DD}}$ (orange) and measured their relative tumor initiation rates over time (Figure 2A). CRE expression activated oncogenic Hras ${ }^{G 12 V}$ along with YFP expression in transduced cells and mice with transduced epidermis were born asymptomatic and they developed normally after birth. However, some $\mathrm{Hras}^{G 12 V}$ expressing mice began to develop tumors after $40+$ days (Figure 2B). Ectopic F-SOX2 ${ }^{\mathrm{WT}}$ or F-SOX2 ${ }^{\mathrm{AA}}$ expression significantly enhanced the rate of tumor initiation in this $\mathrm{HRAS}^{\mathrm{G} 12 \mathrm{~V}}$ driven carcinogenesis model. However, this 
accelerated tumor initiation rate was significantly reduced in F-SOX2 ${ }^{\mathrm{DD}}$ expressing mice. Similarly, ectopic Hras ${ }^{G 12 V}$ expression in FACS isolated interfollicular ( $a 6^{\text {hi }}$, Sca ${ }^{\text {hi }}$ ) skin epithelial cells (Figure S2A), resulted in the development of some slow growing and often spontaneously regressing tumors after intra-dermal transplantation. However, if we co-transduced these cells with F-SOX2 ${ }^{\mathrm{WT}}$ or F-SOX2 ${ }^{\mathrm{AA}}$ we measured robust and significantly accelerated tumor growth, whereas $\mathrm{HRAS}^{\mathrm{G} 12 \mathrm{~V}} ; \mathrm{F}-\mathrm{SOX} 2^{\mathrm{DD}}$ expressing tumors expanded significantly slower (Figure 2C, S2B). These data suggest ectopic SOX2 expression promotes HRAS ${ }^{G 12 V}$ driven squamous carcinogenesis in skin, and this activity is reduced in phosphorylation mimetic $S O X 2^{D D}$ mutants.

\section{De novo SOX2 expression inhibits squamous differentiation gene expression.}

Although differential gene expression profiles between DMBA initiated control and Sox2 knock-out SCCs identified a SOX2 regulated gene set in established SCCs (Boumahdi et al., 2014), it is still unclear how de novo SOX2 expression promotes SCC initiation and whether and how SOX2 phosphorylation influences the process. Therefore, we isolated lineage marked cells from $\mathrm{Hras}^{\mathrm{G12V}}$, Hras ${ }^{G 12 V} ; \mathrm{F}-S O X 2^{W T}$, Hras ${ }^{G 12 V} ; F-S O X 2^{A A}$, and $\mathrm{Hras}^{G 12 V} ; F-S O X 2^{D D}$ expressing tumors and determined their gene expression profiles by RNA-seq (Figure S2C). Principle component analyses revealed that $\mathrm{Hras}^{G 12 V} ; \mathrm{F}-S O X 2^{W T}$ and $\mathrm{Hras}^{G 12 V} ; F-S O X 2^{A A}$ expressing tumors are closely related to one another and they are distinct from $\mathrm{Hras}^{G 12 V}$ as well as $\mathrm{Hras}^{G 12 V}$; $F$ $S O X 2^{D D}$ expressing tumors (Figure 2D) consistent with their tumor growth data (Figure 2C). Intriguingly, one $\mathrm{Hras}^{G 12 V}$ tumor was closely related to $\mathrm{Hras}^{G 12 V} ; \mathrm{F}-S O X 2^{W T}$ and Hras ${ }^{G 12 V}$; F$S O X 2^{A A}$ tumors, and closer data inspection revealed that it expressed SOX2 de novo, while SOX2 was not detected in the other two Hras ${ }^{G 12 V}$ tumors (Figure S2D). Confocal immunofluorescence microscopy confirmed SOX2 expression and phosphorylation in this $\mathrm{Hras}^{G 12 V}$ tumor (Figure S2E). This serendipitous finding validates our ectopic SOX2 transgene expression approach as pathologically relevant and powerful to dissect early stages of SCC initiation and promotion.

Differential gene expression analyses between $\mathrm{Hras}^{G 12 V}$ and $\mathrm{Hras}^{G 12 V}$;F-SOX2 ${ }^{W T}$ driven tumors revealed SOX2 inhibits the tumor suppressive NOTCH (Jag1, Rbpj) (Alcolea et al., 2014; Fortunel et al., 2003; Rangarajan et al., 2001; South et al., 2014) and TGF $\beta$ (Tgfb1, Tgfbr1, Tgfbr2, Smurf1) (Guasch et al., 2007; White et al., 2010) signaling pathways along with spinous differentiation ( $K r t 1$ and $K r t 10$ ) markers (Figure 2E). In addition, we uncovered a significant increase in TPC marker expression (Cd34 (Malanchi et al., 2008), Vegfc (Beck et al., 2011; Lichtenberger et al., 2010), Nrp1 (Beck et al., 2011; Siegle et al., 2014), and Twist1 (Beck et al., 2015)) and suppression of squamous differentiation genes (KIf4 (Fuchs et al., 1999; Li et al., 2019), Grh/3 (Yu et al., 2006), Dsg1, Dsg2, Dsc1, Dsc2 (Dusek et al., 2007), Dlx3 (Morasso et 
al., 1996; Palazzo et al., 2016) and Tgm1, Tgm3 (Eckert et al., 2005)) in $\operatorname{Hras}^{G 12 V}$;F-SOX2 ${ }^{A A}$ compared to Hras ${ }^{G 12 V} ; F-S O X 2^{D D}$ SCCs (Figure 2F). These data suggest SOX2 promotes tumor initiation by suppressing squamous differentiation and this activity is inhibited by SOX2 phosphorylation.

\section{SOX2-phosphorylation inhibits progenitor cell self-renewal, SCC initiation and growth.}

Consistent with the idea that SOX2 phosphorylation inhibits TPC self-renewal and promotes their differentiation, we detected higher KLF4 expression in $\mathrm{Hras}^{G 12 V}$;F-SOX2 ${ }^{D D}$ compared to Hras $^{G 12 V}$;F-SOX2 ${ }^{A A}$ SCCs (Figure 2G). In addition, FACS analyses revealed a significantly reduced fraction of undifferentiated $\left(a 6^{\text {hi }} \beta^{\text {hi }}\right)$ SCC cells alongside a larger cohort of differentiated $\left(a 6^{\text {lo }} \beta 1^{\text {lo }}\right.$ ) SCC cells in $\operatorname{Hras}^{G 12 V}$;F-SOX2 $2^{D D}$ tumors (Figure 2H, S2C). To measure TPC selfrenewal rates, we also labeled cycling cells with EdU (5-Ethynyl-2'deoxyuridine) for $2 \mathrm{hrs,} \mathrm{followed}$ by an $8 \mathrm{hr}$ BrdU (5-Bromo-2'deoxyuridine) pulse (Figure S2F). Next, we determined the fraction of undifferentiated cells that stained positive for EdU but not KRT10, over all EdU labelled cells, including KRT10 positive, differentiated SCC cells with confocal microscopy (Ying et al., 2018). These experiments also suggested TPC self-renewal is significantly reduced in Hras ${ }^{G 12 V}$; $F$ SOX $2^{D D}$ compared to Hras ${ }^{G 12 V} ; F-S O X 2^{A A}$ SCCs (Figure 2I) and SOX2 phosphorylation inhibits TPC self-renewal by accelerating their differentiation into post-mitotic SCC cells.

\section{SOX2-phosphorylation allows TPCs to differentiate in established SCCs.}

To test if SOX2 phosphorylation also effects SCC maintenance, we turned to dimethylbenzanthracene (DMBA) initiated SCCs, which mirror the genetic heterogeneity of human SCCs (Nassar et al., 2015) and are Sox2 dependent (Boumahdi et al., 2014; Siegle et al., 2014). We isolated TPCs from these tumors, transduced them with either F-SOX $2^{W T}$, F$S O X 2^{A A}$, or $F-S O X 2^{D D}$ transgenes before we deleted endogenous Sox2 with CRISPR-Cas9 (Figure 3A). Next, we selected single cell clones and verified Sox2 deletion and F-SOX2 transgene expression (Figure 3B). Western blotting with SOX2 antibodies detected F-SOX2 ${ }^{\mathrm{WT}}$, F-SOX2 $2^{\mathrm{AA}}$, or F-SOX2 ${ }^{\mathrm{DD}}$ transgene expression, but no endogenously expressed SOX2. Furthermore, pS251-SOX2 antibodies identified F-SOX2 ${ }^{\mathrm{WT}}$ and $\mathrm{F}-\mathrm{SOX} 2^{\mathrm{DD}}$, but not F-SOX2 ${ }^{\mathrm{AA}}$ in these TPC clones. We recovered Sox $2^{K O} ; F-S O X 2^{A A}$ clones at a higher frequency compared to Sox $2^{K O} ; F-S O X 2^{W T}$ or Sox $2^{K O} ; F-S O X 2^{D D}$ clones consistent with the idea that unphosphorylated SOX2 is more active in TPCs (Figure S3A).

When we transplanted these clones into mouse dermis, they formed SCCs in which SOX2 was either unphosphorylated or constitutive SOX2 phosphorylation was simulated (Figure 3C). 
We noticed $F-S O X 2^{D D}$ was consistently higher expressed than $F-S O X 2^{A A}$ in our rescued Sox $2^{K O}$ clones. This expression difference suggests phosphorylated SOX2 is less active but increased expression might partially compensate for this activity loss. Nevertheless, Sox $2^{K O} ; F-S O X 2^{D D}$ SCCs had a more differentiated appearance than Sox $2^{K O} ; F-S O X 2^{A A}$ tumors (Figure 3D) and their differential gene expression profiles featuring increased cornification, adhesion, TGF $\beta$ - and NOTCH-activation along with reduced Oxidation/Reduction (Figure 3E) similar to what we observed in our tumor initiation model (Figure 2F).

This increase in squamous differentiation and cornification was also reflected in a significantly higher FLG2 expression (Figure 3F) along with a reduced fraction of undifferentiated ( $a 6^{\text {hi }} / \beta 1^{\text {hi }}$ ) and an increased fraction of differentiated $\left(a 6^{l o} / \beta 1^{\text {lo }}\right)$ SCC cells in Sox $2^{K O} ; F-S O X 2^{D D}$ compared to Sox $2^{K O} ; F-S O X 2^{A A}$ SCCs (Figure 3G-H, S3B). To functionally test if these changes in tumor composition and differentiation affect the tumorigenic SCC cell fraction, we isolated an equal number of lineage-marked Sox $2^{K O} ; F-S O X 2^{A A}$ and Sox $2^{K O} ; F-S O X 2^{D D}$ cells and re-transplanted them in a limited dilution series into mouse dermis. Sox $2^{K O} ; F-S O X 2^{D D}$ SCC cell transplants initiated tumors at a lower frequency (Figure 3I) and a significantly slower rate (Figure 3J) when compared to Sox $2^{K O} ; F-S O X 2^{A A}$ SCCs. These data suggest phosphorylation constrains SOX2 activity, resulting in reduced TPC self-renewal and SCC growth due to increased differentiation, but it doesn't completely inhibit SOX2 function.

\section{Un-phosphorylated and phospho-mimetic SOX2 show similar chromatin binding patterns}

To investigate how SOX2-phosphorylation inhibits TPC self-renewal, we prepared nuclear chromatin from $\operatorname{Hras}^{G 12 V} ; F-S O X 2^{W T}, \operatorname{Hras}^{G 12 V} ; F-S O X 2^{A A}$, and $\operatorname{Hras}^{G 12 V} ; F-S O X 2^{D D}$, as well as Sox $2^{K O} ; F-S O X 2^{W T}$, Sox $2^{K O} ; F-S O X 2^{A A}$, and Sox $2^{K O} ; F-S O X 2^{D D}$ SCC models for chromatin immunoprecipitation sequencing (ChIP-seq). To accurately normalize these ChIP-seq data, we spiked equal amounts of chromatin we isolated from a human SCC cell line into each murine ChIP-seq sample before immuno-precipitation. After each sample was sequenced, we aligned mouse and human sequences that co-precipitated with SOX2 against the respective reference genomes and normalized mouse relative to their corresponding human sequence counts. Although the DNA-binding ability of transcription factors is often inhibited when they become phosphorylated, we found F-SOX2 ${ }^{\mathrm{WT}}$, F-SOX2 ${ }^{\mathrm{AA}}$ and F-SOX2 ${ }^{\mathrm{DD}}$ ChIP-seq profiles were highly concordant and more than $80 \%$ of SOX2-peaks contained the SOX-family motif at their peak summits (Figure 4A-B). These data suggest our ChIP-seq results are specific and SOX2phosphorylation does not globally interfere with its DNA-binding ability. 
However, differential read count analyses identified a small subset of cis-regulatory elements that were preferentially F-SOX2 $2^{\mathrm{AA}}$ or F-SOX2 ${ }^{\mathrm{DD}}$ bound (Figure $4 \mathrm{~A}-\mathrm{B}$ ). We used the genomic regions enrichment of annotations tool (GREAT, (McLean et al., 2010)) to identify genes with a transcriptional start site within $1 \mathrm{Mb}$ of each SOX2 binding site and intersected them with genes that are consistently up- or down-regulated in $\mathrm{Hras}^{\mathrm{G12V}} ; \mathrm{F}-S O X 2^{A A}$ and $S o x 2^{K O} ; F-S O X 2^{A A}$ compared to $\operatorname{Hras}^{G 12 V} ; \mathrm{F}-S O X 2^{D D}$ and Sox $2^{K O} ; F-S O X 2^{D D}$ tumors, respectively. Surprisingly, this approach identified only four genes that were inhibited and four genes that were activated by increased SOX2 $2^{\mathrm{AA}}$ compared to SOX2 ${ }^{\mathrm{DD}}$ binding (Figure 4C). KIf4 emerged as the gene with the highest average expression, and it was consistently suppressed in SOX $2^{\mathrm{AA}}$ compared to SOX $2^{\mathrm{DD}}$ SCCs in both of our tumor models (Figure 4D). Quantitative confocal immuno-fluorescence microscopy confirmed KLF4 expression is significantly increased in basal and suprabasal Sox $2^{K O} ; F-S O X 2^{D D}$ compared to Sox $2^{K O} ; F-S O X 2^{A A}$ SCC cells (Figure 4E-F), similar to what we observed in $\mathrm{Hras}^{\mathrm{G} 12 \mathrm{~V}}$;F-SOX2 ${ }^{D D}$ compared to $\mathrm{Hras}^{\mathrm{G} 12 \mathrm{~V}}$;F-SOX2 ${ }^{A A}$ tumors (Figure 2F).

\section{Phosphorylated SOX2 is evicted from a specific KIf4 enhancer to permit KLF4 binding and increased Klf4 expression}

Our ChIP-seq and differential expression data suggest SOX2 inhibits KIf4 transcription, but its expression can be restored when SOX2 becomes phosphorylated in SCC cells. Indeed, our differential SOX2 ChIP-seq analyses identified F-SOX2 ${ }^{\mathrm{AA}}$ but not F-SOX2 ${ }^{\mathrm{DD}}$ on a large enhancer cluster (EC944) located $944 \mathrm{~kb}$ distal to the KIf4 transcriptional start site (TSS) (Figure 5A). H3K27Ac ChIP-seq also indicated that this enhancer is more active in Sox $2^{K O}$; SOX $2^{D D}$ compared to Sox $2^{K O}$; SOX $2^{A A}$ SCCs and we found Mediator (Med12) enrichment at this site, suggesting promoter-enhancer interactions. To test this hypothesis, we used chromatin conformation capture sequencing (4C-seq) with a bait located at the KIf4 TSS to unbiasedly search for promoterenhancer interactions (Figure 5A). 4C-ker (Raviram et al., 2016) identified statistically significant interactions between the Klf4 promoter and several cis-regulatory elements including KIf4 ${ }^{E C 944}$ and $K I f 4^{E C 390}$, which we previously identified as a potential SOX2 and PITX1 bound KIf4 enhancer (Sastre-Perona et al., 2019). However, Klf4 ${ }^{E C 390}$ was, in contrast to $K / f 4^{E C 944}$, equally bound by $\mathrm{SOX} 2^{\mathrm{AA}}$ and $\mathrm{SOX} 2^{\mathrm{DD}}$.

To functionally test if EC944 regulates KIf4 transcription, we designed two independent gRNAs that targeted either a SOX2 or KLF4 motif located at the peak summit (Figure S4A). A gRNA directed against tdTomato $\left(g R N A^{T o m}\right)$ served as control. Induction of HA-dCas9-KRAB with doxycycline significantly reduced KIf4 transcription when it was targeted to KIf4 ${ }^{E C 944}$ compared to gRNA ${ }^{\text {Tom }}$ or two gRNAs targeting SOX2 and KLF4 bound sites at EC1001 (Figure 5B-C). To 
further characterize $K_{I f 4^{E C 944}}$ we cloned it into a fluorescent activity reporter (Figure 5D). Next, we transduced TPCs with this reporter, transplanted them into mouse dermis and assessed the expression of KIf4 ${ }^{E C 944}$ driven GFP expression within transduced cells that constitutively expressed H2B-RFP. FACS analyses distinguished cells with low, medium and high GFP expression within the RFP ${ }^{+}$-lineage and their GFP expression levels correlated inversely with a6and $\beta 1$-integrin levels (Figure 5E). Furthermore, SOX2 expression declined with increasing GFP expression, whereas KLF4 levels transiently increased (Figure 5F). Consistent with these data we detected only a few basal SCC cells with weak GFP expression, while GFP was highly expressed in supra-basal SCC layers (Figure 5G) and RFPhi/GFPlow SCC-cells initiated tumors more efficiently than RFPhi/GFP ${ }^{\text {hi }}$ SCC-cells, when they were transplanted into mouse dermis (Figure 5H). Collectively, these data suggest that SOX2 binds KIf4 ${ }^{E C 944}$ to repress KIf4 transcription in TPCs, but when SOX2 is phosphorylated, it is specifically evicted from this site resulting in increased KLF4 expression and squamous differentiation.

Because KIf4 ${ }^{E C 944}$ contains two KLF4 motifs directly adjacent to SOX2 and PITX1 motifs (Figure S4A), we wondered whether the eviction of SOX2 upon phosphorylation would allow KLF4 to occupy this enhancer and convert it from a transcriptional repressor into an activator. To test this hypothesis, we isolated $a 6^{\mathrm{hi}} / \beta 1^{\mathrm{hi}}$ and $a 6^{\mathrm{lo}} / \beta 1^{\mathrm{lo}}$ cells from lineage marked $S O X 2^{K O} ; F$ $S O X 2^{A A}$ and SOX ${ }^{K O} ; F-S O X 2^{D D}$ SCCs by FACS (Figure S4B) and determined the occupancy of KLF4 in these cells with ChIP-seq. We found KLF4 is hardly detected on KIf4 ${ }^{E C 944}$ in a6 ${ }^{\text {hi }} / \beta 1^{\text {hi }}$ $S O X 2^{K O} ; F-S O X 2^{A A} S C C$ cells, but it was significantly enriched this site in a $6^{\text {hi }} / \beta 1^{\text {hi }} S O X 2^{K O} ; F-$ $S O X 2^{D D}$ SCC cells (Figure 5A). KLF4 was also detected at comparable levels in $a 6^{\mathrm{lo}} / \beta 1^{\mathrm{lo}}$ cells of $S O X 2^{K O} ; F-S O X 2^{A A}$ and SOX $2^{K O} ; F-S O X 2^{D D}$ SCCs. These data suggest that SOX2 and KLF4 bind to KIf4 ${ }^{E C 944}$ in a mutually exclusive manner, SOX2 dominates over KLF4 in TPCs, and it inhibits KIf4 transcription and KLF4 dependent differentiation. However, when SOX2 becomes phosphorylated, it is evicted from KIf4 ${ }^{E C 944}$, which allows KLF4 to bind, activate the enhancer, and boost KLF4 expression and squamous differentiation.

\section{Increased KLF4 expression and chromatin binding promotes epidermal differentiation}

As a result of increased KLF4 expression, we detected significantly more chromatin bound KLF4 in both $a 6^{\mathrm{hi}} / \beta 1^{\mathrm{hi}}$ and $\mathrm{a} 6^{\mathrm{lo}} / \beta 1^{\mathrm{lo}}$ fractions of $S O X 2^{K O} ; F-S O X 2^{D D}$ compared to $S O X 2^{K O} ; F$ SOX $2^{A A}$ SCCs with ChIP-seq (Figure 6A). De novo motif predictions identified KLF4-like motifs in $>60 \%$ of KLF4 ChIP-seq peaks at their peak summits (Figure 6A, S4C). Although differential KLF4 binding data revealed 290 sites with KLF4 enrichment in $a 6^{\text {hi }} / \beta 1^{\text {hi }}$ cells of $S O X 2^{K O} ; S O X 2^{A A}$ compared to $S O X 2^{K O} ; S O X 2^{D D}$ SCCs, its enrichment was rather modest. In contrast, we identified 
317 sites that were enriched in $a 6^{\text {hi }} / \beta 1^{\text {hi }}$ cells of $S O X 2^{K O} ; S O X 2^{D D}$ compared to $S O X 2^{K O} ; S O X 2^{A A}$ SCCs with an $\sim 3$-fold median enrichment in chromatin binding (Figure 6B). Intersection of these KLF4 bound sites with transcripts that are significantly up-regulated in Sox $2^{K O} ; S O X 2^{A A}$ or Sox $2^{\mathrm{KO}} ; \mathrm{SOX} 2^{\mathrm{DD}}$ SCC cells (Figure $6 \mathrm{C}$ ) revealed 138 genes that are directly upregulated as a response of increased KLF4 expression in Sox $2^{K O} ; S O X 2^{D D}$ SCCs. Amongst these KLF4 targets we found KIf4 itself along with genes that promote epidermal differentiation, keratinization, and barrier formation (Figure 6D-E). Conversely, we also noticed increased KLF4 enrichment on the SCC specific SOX2 enhancer in SOX2 ${ }^{\mathrm{DD}}$ mutant SCCs. These data suggest KLF4 drives the expression of squamous differentiation genes (Figure 6E) and it feeds back and occupies the KLF4-motif in the SCC specific Sox2 enhancer (Figure 6F,G) to inhibit SOX2 expression (SastrePerona et al., 2019) and stabilize the differentiated, post-mitotic SCC-cell state.

\section{Discussion}

The fate choice between stem cell self-renewal and differentiation is regulated by bistable transcriptional networks that allow cells to stably reside in one of two alternative cell states (Davis and Rebay, 2017; Young, 2011). Genetic gain- and loss-of-function studies identified master regulatory transcription factors as the core components of these networks and they found these transcription factors promote self-renewal by enhancing each other's expression in positive feedforward circuits, while they repress transcriptional programs that promote differentiation. Conversely, differentiation promoting transcription factors enhance their own expression as they inhibit the self-renewal network. We and others previously identified several components of a bistable transcriptional network that governs self-renewal and differentiation in SCCs (Boumahdi et al., 2014; Sastre-Perona et al., 2019; Siegle et al., 2014). We found this network is comprised of mutually exclusive SOX2-PITX1- $\triangle$ Np63 self-renewal and KLF4 dependent differentiation circuits (Sastre-Perona et al., 2019). Likewise, a cancer driving SOX2- $\Delta$ Np63-KLF5 feed-forward circuit has been described in human esophageal SCCs (Jiang et al., 2018, 2020; Watanabe et al., 2014). Although new components of this SCC regulatory bistable transcriptional network are being identified, it remained unclear how TPCs can differentiate in steady state tumors, if KLF4 controls the switch from self-renewal to differentiation, but its expression is blocked in selfrenewing TPCs.

In this study we identified a molecular mechanism that resolves this self-renewal differentiation paradox. We discover that SOX2 - a core component of the self-renewal circuit can get phosphorylated in TPCs and increased SOX2 phosphorylation reduces TPC self-renewal and it enables them to differentiate into post-mitotic SCC cells (Figure 6G). Although we found 
that changes in SOX2 phosphorylation affected TPC self-renewal and differentiation rates and that these changes had a profound impact on SCC initiation and tumor growth, we found no changes in cell proliferation and survival programs that have been linked to SOX2 in SCCs (Boumahdi et al., 2014). This finding is consistent with a prior study that found cyclin dependent kinase mediated SOX2 phosphorylation of S37 and S251 enhances the ability of SOX2 to induce a pluripotent embryonic stem cell (ESC) state, and that SOX2 phosphorylation was dispensable for ESC self-renewal (Ouyang et al., 2015). These studies suggest phosphorylated SOX2 has highly specific and context dependent functions, and a better understanding of their roles and regulation could inform the development of differentiation therapies for patients with SCCs or other SOX2 regulated cancers like glioblastoma or astrocytoma (Modrek et al., 2017; Suvà et al., 2014).

Although several post-translational SOX2 modifications have been reported, their roles and regulation are context specific and poorly understood (Williams et al., 2019). While human SOX2 S37 and S251 phosphorylation could have simply affected nuclear import or export, reduced SOX2 stability, or impaired its DNA binding ability, we found its effects are much more context dependent. Quantitative ChIP-seq studies with non-phosphorylated SOX2 ${ }^{\mathrm{AA}}$ and phosphorylation mimetic SOX2 ${ }^{\mathrm{DD}}$ mutants with spike in controls for data normalization revealed that their DNA binding patterns are highly concordant, they only differed in $\sim 5 \%$ of $\mathrm{SOX} 2^{\mathrm{AA}}$ or SOX $2^{\mathrm{DD}}$ bound sites, where SOX2 phosphorylation increased or decreased SOX2 - DNA interactions dependent on the gene regulatory site. We only identified eight gene regulatory sites that were more strongly bound by SOX $2^{\mathrm{AA}}$ compared to SOX $2^{\mathrm{DD}}$ and that could be linked to consistent changes in gene expression in both of our tumor models. The expression of half of these genes increased in SOX $2^{\mathrm{DD}}$ tumors, while the other genes decreased. No consistent changes in gene expression were observed in genes that were more strongly bound by $\mathrm{SOX} 2^{\mathrm{DD}}$.

Amongst the gene regulatory elements that were bound by SOX2 ${ }^{\mathrm{WT}}$ and $\mathrm{SOX} 2^{\mathrm{AA}}$ but not SOX $2^{\mathrm{DD}}$ we identified an enhancer cluster KIf4 ${ }^{E C 944}$ that is located $944 \mathrm{~kb}$ upstream the KIf4 TSS. Although this enhancer is much closer to Act/7b than to KIf4 in linear distance, we were unable to detect $A c t / 7 b$ in SOX2 ${ }^{\mathrm{AA}}$ or SOX2 $2^{\mathrm{DD}}$ expressing SCCs, while the expression of KIf4 was significantly higher in SOX $2^{\mathrm{DD}}$ compared to SOX $2^{\mathrm{AA}}$ SCCs. Consistent with these changes in gene expression and SOX2 binding, we found the Klf4 promoter interacts with EC944 by 4C-seq. Additionally, the activity of a LV-PGK-H2B-RFP;EC944-GFP expression reporter increases as a6/ $\beta 1$-integrin and SOX2 levels decline, and KLF4 expression strengthens while cells transition from basal- to suprabasal SCC layers. These data suggest SOX2 binds to EC944 to inhibit KIf4 
transcription in TPCs, but SOX2 becomes evicted from this site when it is phosphorylated and this allows residual KLF4 to bind this site to enhance its own expression. Once KLF4 expression increases it begins to accumulate at many gene regulatory elements across the epigenome to promote the expression of squamous differentiation markers and inhibit TPC self-renewal and proliferation.

Although this study uncovers a novel gene regulatory mechanism that can resolve the selfrenewal differentiation paradox, it also reveals that de novo expressed SOX2 promotes clonal expansion and SCC growth partly because it can directly inhibit the expression of several TGF $\beta$ and Notch-pathway components with known tumor suppressive functions in stratified epithelial cells. However, our study also raises new questions. One of the most exciting emerging questions is why phosphorylated SOX2 gets evicted from KIf4 ${ }^{E C 944}$, but not from most other SOX2 bound sites? It is intriguing to speculate that the addition of negatively charged phosphate groups could introduce structural changes that perturb the interaction of SOX2 with other transcription factors or transcriptional co-regulators. These changes could weaken their protein-DNA interactions and it may allow residual KLF4 to displace SOX2 from sites that are rich in KLF4 binding motifs. Indeed, KIf4 ${ }^{E C 944}$ contains central PITX- and SOX-motifs that are surrounded by several KLFmotifs and it is possible that SOX2 and KLF4 competitively occupy this site, where their binding kinetics would depend on the active concentrations of SOX2, KLF4, and their respective transcriptional partners.

Another emerging question is which kinases and phosphatases are responsible for the phosphorylation of SOX2 in SCCs. In vitro studies suggested that S37 and S251 of human SOX2 can be phosphorylated by CDK2, Aurora kinase A, or MAPK1/3 (Ji et al., 2018; Lim et al., 2017; Ouyang et al., 2015), but it remains to be tested whether SOX2 is a substrate of cell cycle regulated or rather transcriptional CDKs in SCCs. Even more exciting than the identification of the SOX2 regulatory kinases would be the identification of SOX2 regulatory phosphatases as their inhibition would increase SOX2 phosphorylation and squamous differentiation in tumors thus inhibiting SCC growth. Although these questions remain to be answered in future studies, our data suggest that SOX2 may cycle between phosphorylated and un-phosphorylated states and this cycling behavior along with the competitive occupancy of KIf4EC944 by SOX2 or KLF4 may explain why the fate choice between self-renewal and differentiation is stochastically regulated (Driessens et al., 2012) and how cell fate decissions can become biased towards self-renewal or differentiation dependent on the effective concentration of SOX2 and KLF4, respectively. 


\section{Acknowledgments}

We thank E. Fuchs and P. Khavari for reagents, Ramya Raviram and Sana Badri for computational advice, and Eva Gonzalez, Eva Hernando-Monge and Timothee Lionnet for discussions. We are grateful to the NYU Langone Health Flow cytometry core facility, the Division of Comparative Medicine for expert handling and care of mice, the NYU Langone Health genome technology center for next generation sequencing, and the High-Performance Computing Facility for cluster access and data storage. This research was supported by NIH grant 1R01-CA24817501A1, American Cancer Society grant RSG-16-033-01-DDC, Worldwide Cancer and the OrbuchBrand Pilot Grant Program for Cancers of the Skin to M.S., T32 CA009161 and T32 AR064184 to A.S-P. and T32 CA009161 to S.H-P. Core funding was partially supported by NIH grant P30 CA016087 to the Perlmutter Cancer Center.

\section{Author Contributions}

Conceptualization, S.H-P., A.S-P. and M.S.; Methodology, S.H-P., M.A., A.S-P., P.R., Z.Y., B.AO., and M.S.; Formal Analysis, S.H-P., M.A., A.S.-P., Z.Y. and M.S.; Investigation, A.S.-P., S.HP., M.A., J.S., I.A., S.B., and M.S.; Writing-Review and Editing, S.H.P. and M.S.; WritingReview and Editing, S.H-P., A.S-P., Y.Z., S.B. and M.S.; Visualization, S.H-P., A.S-P. and M.S.; Funding Acquisition, M.S.

\section{Declaration of Interests}

The authors declare no competing interest. 


\section{References}

Alcolea, M.P., Greulich, P., Wabik, A., Frede, J., Simons, B.D., and Jones, P.H. (2014). Differentiation imbalance in single oesophageal progenitor cells causes clonal immortalization and field change. Nature Cell Biology 16, 615-622.

Anders, S., Pyl, P.T., and Huber, W. (2015). HTSeq-a Python framework to work with highthroughput sequencing data. Bioinformatics 31, 166-169.

Arnold, K., Sarkar, A., Yram, M.A., Polo, J.M., Bronson, R., Sengupta, S., Seandel, M., Geijsen, N., and Hochedlinger, K. (2011). Sox2(+) adult stem and progenitor cells are important for tissue regeneration and survival of mice. Cell Stem Cell 9, 317-329.

Bailey, T.L., Boden, M., Buske, F.A., Frith, M., Grant, C.E., Clementi, L., Ren, J., Li, W.W., and Noble, W.S. (2009). MEME SUITE: tools for motif discovery and searching. Nucleic Acids Research 37, W202-8.

Beck, B., Driessens, G., Goossens, S., Youssef, K.K., Kuchnio, A., Caauwe, A., Sotiropoulou, P.A., Loges, S., Lapouge, G., Candi, A., et al. (2011). A vascular niche and a VEGF-Nrp1 loop regulate the initiation and stemness of skin tumours. Nature 478, 399-403.

Beck, B., Lapouge, G., Rorive, S., Drogat, B., Desaedelaere, K., Delafaille, S., Dubois, C., Salmon, I., Willekens, K., Marine, J.-C., et al. (2015). Different Levels of Twist1 Regulate Skin Tumor Initiation, Stemness, and Progression. Cell Stem Cell 16, 67-79.

Beronja, S., Livshits, G., Williams, S., and Fuchs, E. (2010). Rapid functional dissection of genetic networks via tissue-specific transduction and RNAi in mouse embryos. Nat Med 16, 821-827.

Blanpain, C., and Simons, B.D. (2013). Unravelling stem cell dynamics by lineage tracing. Nat Rev Mol Cell Biol 14, 489-502.

Boumahdi, S., Driessens, G., Lapouge, G., Rorive, S., Nassar, D., Mercier, M.L., Delatte, B., Caauwe, A., Lenglez, S., Nkusi, E., et al. (2014). SOX2 controls tumour initiation and cancer stem-cell functions in squamous-cell carcinoma. Nature 511, 246-250.

Carbon, S., Ireland, A., Mungall, C.J., Shu, S., Marshall, B., Lewis, S., Hub, A., and Group, W.P.W. (2009). AmiGO: online access to ontology and annotation data. Bioinformatics 25, 288289.

Chen, X., Mitsutake, N., LaPerle, K., Akeno, N., Zanzonico, P., Longo, V.A., Mitsutake, S., Kimura, E.T., Geiger, H., Santos, E., et al. (2009). Endogenous expression of HrasG12V induces developmental defects and neoplasms with copy number imbalances of the oncogene. Proc National Acad Sci 106, 7979-7984. 
Davis, T.L., and Rebay, I. (2017). Master regulators in development: Views from the Drosophila retinal determination and mammalian pluripotency gene networks. Developmental Biology 421, 93-107.

Driessens, G., Beck, B., Caauwe, A., Simons, B.D., and Blanpain, C. (2012). Defining the mode of tumour growth by clonal analysis. Nature $488,1-5$.

Dusek, R.L., Godsel, L.M., and Green, K.J. (2007). Discriminating roles of desmosomal cadherins: Beyond desmosomal adhesion. Journal of Dermatological Science 45, 7-21.

Eckert, R.L., Sturniolo, M.T., Broome, A.-M., Ruse, M., and Rorke, E.A. (2005).

Transglutaminase Function in Epidermis. Journal of Investigative Dermatology 124, 481-492.

Ezhkova, E., Lien, W.-H., Stokes, N., Pasolli, H.A., Silva, J.M., and Fuchs, E. (2011). EZH1 and $\mathrm{EZH} 2$ cogovern histone H3K27 trimethylation and are essential for hair follicle homeostasis and wound repair. Genes Dev 25, 485-498.

Feng, J., Liu, T., Qin, B., Zhang, Y., and Liu, X.S. (2012). Identifying ChIP-seq enrichment using MACS. Nature Protocols 7, 1728-1740.

Fortunel, N.O., Otu, H.H., Ng, H.-H., Chen, J., Mu, X., Chevassut, T., Li, X., Joseph, M., Bailey, C., Hatzfeld, J.A., et al. (2003). Comment on "'Stemness': transcriptional profiling of embryonic and adult stem cells" and "a stem cell molecular signature". Science 302, 393-author reply 393.

Fuchs, E., Segre, J.A., and Bauer, C. (1999). Klf4 is a transcription factor required for establishing the barrier function of the skin. Nat Genet 22, 356-360.

Ge, Y., Gomez, N.C., Adam, R.C., Nikolova, M., Yang, H., Verma, A., Lu, C.P.-J., Polak, L., Yuan, S., Elemento, O., et al. (2017). Stem Cell Lineage Infidelity Drives Wound Repair and Cancer. Cell 169, 636-642.e14.

Guasch, G., Schober, M., Pasolli, H.A., Conn, E.B., Polak, L., and Fuchs, E. (2007). Loss of TGF $\beta$ Signaling Destabilizes Homeostasis and Promotes Squamous Cell Carcinomas in Stratified Epithelia. Cancer Cell 12, 313-327.

Hulsen, T., Vlieg, J. de, and Alkema, W. (2008). BioVenn - a web application for the comparison and visualization of biological lists using area-proportional Venn diagrams. BMC Genomics 9 , 488.

Ji, J., Yu, Y., Li, Z.-L., Chen, M.-Y., Deng, R., Huang, X., Wang, G.-F., Zhang, M.-X., Yang, Q., Ravichandran, S., et al. (2018). XIAP Limits Autophagic Degradation of Sox2 and Is A Therapeutic Target in Nasopharyngeal Carcinoma Stem Cells. Theranostics 8, 1494-1510.

Jiang, Y., Jiang, Y.-Y., Xie, J.-J., Mayakonda, A., Hazawa, M., Chen, L., Xiao, J.-F., Li, C.-Q., Huang, M.-L., Ding, L.-W., et al. (2018). Co-activation of super-enhancer-driven CCAT1 by TP63 and SOX2 promotes squamous cancer progression. Nat Commun 9, 3619.

Jiang, Y.-Y., Jiang, Y., Li, C.-Q., Zhang, Y., Dakle, P., Kaur, H., Deng, J.-W., Lin, R.Y.-T., Han, L., Xie, J.-J., et al. (2020). TP63, SOX2, and KLF5 Establish a Core Regulatory Circuitry That 
Controls Epigenetic and Transcription Patterns in Esophageal Squamous Cell Carcinoma Cell Lines. Gastroenterology 159, 1311-1327.e19.

Kim, D., Pertea, G., Trapnell, C., Pimentel, H., Kelley, R., and Salzberg, S.L. (2013). TopHat2: accurate alignment of transcriptomes in the presence of insertions, deletions and gene fusions. Genome Biology 14, R36.

Kinoshita, E., Kinoshita-Kikuta, E., Takiyama, K., and Koike, T. (2006). Phosphate-binding Tag, a New Tool to Visualize Phosphorylated Proteins. Molecular \& Cellular Proteomics 5, 749-757.

Klein, A.M., and Simons, B.D. (2011). Universal patterns of stem cell fate in cycling adult tissues. Development (Cambridge, England) 138, 3103-3111.

Klein, A.M., Brash, D.E., Jones, P.H., and Simons, B.D. (2009). Stochastic fate of p53-mutant epidermal progenitor cells is tilted toward proliferation by UV B during preneoplasia.

Proceedings of the National Academy of Sciences 107, 270-275.

Kreso, A., and Dick, J.E. (2014). Evolution of the Cancer Stem Cell Model. Cell Stem Cell 14, 275-291.

Langmead, B., and Salzberg, S.L. (2012). Fast gapped-read alignment with Bowtie 2. Nat Methods 9, 357-359.

Li, H., Handsaker, B., Wysoker, A., Fennell, T., Ruan, J., Homer, N., Marth, G., Abecasis, G., Durbin, R., and Subgroup, 1000 Genome Project Data Processing (2009). The Sequence Alignment/Map format and SAMtools. Bioinformatics 25, 2078-2079.

Li, J., Zheng, H., Yu, F., Yu, T., Liu, C., Huang, S., Wang, T.C., and Ai, W. (2012). Deficiency of the Kruppel-like factor KLF4 correlates with increased cell proliferation and enhanced skin tumorigenesis. Carcinogenesis 33, 1239-1246.

Li, X.M., Kim, S.J., Hong, D.-K., Jung, K.E., Choi, C.W., Seo, Y.-J., Lee, J.-H., Lee, Y., and Kim, C.-D. (2019). KLF4 suppresses the tumor activity of cutaneous squamous cell carcinoma (SCC) cells via the regulation of SMAD signaling and SOX2 expression. Biochemical and Biophysical Research Communications 516, 1110-1115.

Lichtenberger, B.M., Tan, P.K., Niederleithner, H., Ferrara, N., Petzelbauer, P., and Sibilia, M. (2010). Autocrine VEGF signaling synergizes with EGFR in tumor cells to promote epithelial cancer development. Cell 140, 268-279.

Lim, S., Bhinge, A., Alonso, S.B., Aksoy, I., Aprea, J., Cheok, C.F., Calegari, F., Stanton, L.W., and Kaldis, P. (2017). Cyclin-Dependent Kinase-Dependent Phosphorylation of Sox2 at Serine 39 Regulates Neurogenesis. Mol Cell Biol 37, 1409-1424.

Love, M.I., Huber, W., and Anders, S. (2014). Moderated estimation of fold change and dispersion for RNA-seq data with DESeq2. Genome Biology 15, 550.

Machanick, P., and Bailey, T.L. (2011). MEME-ChIP: motif analysis of large DNA datasets. Bioinformatics 27, 1696-1697. 
Malanchi, I., Peinado, H., Kassen, D., Hussenet, T., Metzger, D., Chambon, P., Huber, M., Hohl, D., Cano, A., Birchmeier, W., et al. (2008). Cutaneous cancer stem cell maintenance is dependent on $\beta$-catenin signalling. Nature 452, 650-653.

McLean, C.Y., Bristor, D., Hiller, M., Clarke, S.L., Schaar, B.T., Lowe, C.B., Wenger, A.M., and Bejerano, G. (2010). GREAT improves functional interpretation of cis-regulatory regions. Nat Biotechnol 28, 495-501.

Modrek, A.S., Golub, D., Khan, T., Bready, D., Prado, J., Bowman, C., Deng, J., Zhang, G., Rocha, P.P., Raviram, R., et al. (2017). Low-Grade Astrocytoma Mutations in IDH1, P53, and ATRX Cooperate to Block Differentiation of Human Neural Stem Cells via Repression of SOX2. Cell Reports 21, 1267-1280.

Morasso, M.I., Markova, N.G., and Sargent, T.D. (1996). Regulation of epidermal differentiation by a Distal-less homeodomain gene. Journal of Cell Biology 135, 1879-1887.

Nassar, D., Latil, M., Boeckx, B., Lambrechts, D., and Blanpain, C. (2015). Genomic landscape of carcinogen-induced and genetically induced mouse skin squamous cell carcinoma. Nat Med $21,946-954$.

Ouyang, J., Yu, W., Liu, J., Zhang, N., Florens, L., Chen, J., Liu, H., Washburn, M., Pei, D., and Xie, T. (2015). Cyclin-dependent kinase-mediated Sox2 phosphorylation enhances the ability of Sox2 to establish the pluripotent state. The Journal of Biological Chemistry 290, 22782-22794.

Palazzo, E., Kellett, M., Cataisson, C., Gormley, A., Bible, P.W., Pietroni, V., Radoja, N., Hwang, J., Blumenberg, M., Yuspa, S.H., et al. (2016). The homeoprotein DLX3 and tumor suppressor p53 co-regulate cell cycle progression and squamous tumor growth. Oncogene 35 , 3114-3124.

Pierce, G.B., and Wallace, C. (1971). Differentiation of malignant to benign cells. Cancer Research 31, 127-134.

Ramírez, F., Dündar, F., Diehl, S., Grüning, B.A., and Manke, T. (2014). deepTools: a flexible platform for exploring deep-sequencing data. Nucleic Acids Research 42, W187-91.

Rangarajan, A., Talora, C., Okuyama, R., Nicolas, M., Mammucari, C., Oh, H., Aster, J.C., Krishna, S., Metzger, D., Chambon, P., et al. (2001). Notch signaling is a direct determinant of keratinocyte growth arrest and entry into differentiation. Embo Journal 20, 3427-3436.

Raviram, R., Rocha, P.P., Müller, C.L., Miraldi, E.R., Badri, S., Fu, Y., Swanzey, E., Proudhon, C., Snetkova, V., Bonneau, R., et al. (2016). 4C-ker: A Method to Reproducibly Identify Genome-Wide Interactions Captured by 4C-Seq Experiments. PLoS Computational Biology 12, e1004780.

Robinson, J.T., Thorvaldsdóttir, H., Winckler, W., Guttman, M., Lander, E.S., Getz, G., and Mesirov, J.P. (2011). Integrative genomics viewer. Nat Biotechnol 29, 24-26. 
Rocha, P.P., Raviram, R., Fu, Y., Kim, J., Luo, V.M., Aljoufi, A., Swanzey, E., Pasquarella, A., Balestrini, A., Miraldi, E.R., et al. (2016). A Damage-Independent Role for 53BP1 that Impacts Break Order and Igh Architecture during Class Switch Recombination. Cell Reports 16, 48-55.

Sastre-Perona, A., Hoang-Phou, S., Leitner, M.-C., Okuniewska, M., Meehan, S., and Schober, M. (2019). De Novo PITX1 Expression Controls Bi-Stable Transcriptional Circuits to Govern Self-Renewal and Differentiation in Squamous Cell Carcinoma. Cell Stem Cell 24, 390-404.e8.

Schmidl, C., Rendeiro, A.F., Sheffield, N.C., and Bock, C. (2015). ChIPmentation: fast, robust, low-input ChIP-seq for histones and transcription factors. Nature Methods 12, 963-965.

Schober, M., and Fuchs, E. (2011). Tumor-initiating stem cells of squamous cell carcinomas and their control by TGF- $\beta$ and integrin/focal adhesion kinase (FAK) signaling. Proc National Acad Sci 108, 10544-10549.

Sen, G.L., Boxer, L.D., Webster, D.E., Bussat, R.T., Qu, K., Zarnegar, B.J., Johnston, D., Siprashvili, Z., and Khavari, P.A. (2012). ZNF750 Is a p63 Target Gene that Induces KLF4 to Drive Terminal Epidermal Differentiation. Developmental Cell 22, 669-677.

Siegle, J.M., Basin, A., Sastre-Perona, A., Yonekubo, Y., Brown, J., Sennett, R., Rendl, M., Tsirigos, A., Carucci, J.A., and Schober, M. (2014). SOX2 is a cancer-specific regulator of tumour initiating potential in cutaneous squamous cell carcinoma. Nat Commun 5, 4511.

Simonis, M., Klous, P., Splinter, E., Moshkin, Y., Willemsen, R., Wit, E. de, Steensel, B. van, and Laat, W. de (2006). Nuclear organization of active and inactive chromatin domains uncovered by chromosome conformation capture-on-chip (4C). Nat Genet 38, 1348-1354.

Simons, B.D., and Clevers, H. (2011). Strategies for homeostatic stem cell self-renewal in adult tissues. Cell 145, 851-862.

South, A.P., Purdie, K.J., Watt, S.A., Haldenby, S., Breems, N. den, Dimon, M., Arron, S.T., Kluk, M.J., Aster, J.C., McHugh, A., et al. (2014). NOTCH1 mutations occur early during cutaneous squamous cell carcinogenesis. J Invest Dermatol 134, 2630-2638.

Suvà, M.L., Rheinbay, E., Gillespie, S.M., Patel, A.P., Wakimoto, H., Rabkin, S.D., Riggi, N., Chi, A.S., Cahill, D.P., Nahed, B.V., et al. (2014). Reconstructing and reprogramming the tumorpropagating potential of glioblastoma stem-like cells. Cell 157, 580-594.

Szigety, K.M., Liu, F., Yuan, C.Y., Moran, D.J., Horrell, J., Gochnauer, H.R., Cohen, R.N., Katz, J.P., Kaestner, K.H., Seykora, J.T., et al. (2020). HDAC3 ensures stepwise epidermal stratification via NCoR/SMRT-reliant mechanisms independent of its histone deacetylase activity. Gene Dev 34, 973-988.

Trapnell, C., Roberts, A., Goff, L., Pertea, G., Kim, D., Kelley, D.R., Pimentel, H., Salzberg, S.L., Rinn, J.L., and Pachter, L. (2012). Differential gene and transcript expression analysis of RNAseq experiments with TopHat and Cufflinks. Nature Protocols 7, 562-578. 
Watanabe, H., Ma, Q., Peng, S., Adelmant, G., Swain, D., Song, W., Fox, C., Francis, J.M., Pedamallu, C.S., Deluca, D.S., et al. (2014). SOX2 and p63 colocalize at genetic loci in squamous cell carcinomas. J Clin Invest 124, 1636-1645.

White, R.A., Malkoski, S.P., and Wang, X.J. (2010). TGF $\beta$ signaling in head and neck squamous cell carcinoma. Oncogene $29,5437-5446$.

Whitmarsh, A.J., and Davis, R.J. (2000). Regulation of transcription factor function by phosphorylation. Cellular and Molecular Life Sciences 57, 1172-1183.

Williams, C.A.C., Soufi, A., and Pollard, S.M. (2019). Post-translational modification of SOX family proteins: key biochemical targets in cancer? Semin Cancer Biol 67, 30-38.

Williams, S.E., Beronja, S., Pasolli, H.A., and Fuchs, E. (2011). Asymmetric cell divisions promote Notch-dependent epidermal differentiation. Nature 470, 353-358.

Yang, X., Boehm, J.S., Yang, X., Salehi-Ashtiani, K., Hao, T., Shen, Y., Lubonja, R., Thomas, S.R., Alkan, O., Bhimdi, T., et al. (2011). A public genome-scale lentiviral expression library of human ORFs. Nat Methods 8, 659-661.

Ying, Z., Sandoval, M., and Beronja, S. (2018). Oncogenic activation of PI3K induces progenitor cell differentiation to suppress epidermal growth. Nature Cell Biology 20,1-16.

Young, R.A. (2011). Control of the embryonic stem cell state. Cell 144, 940-954.

Yu, Z., Lin, K.K., Bhandari, A., Spencer, J.A., Xu, X., Wang, N., Lu, Z., Gill, G.N., Roop, D.R., Wertz, P., et al. (2006). The Grainyhead-like epithelial transactivator Get-1/Grhl3 regulates epidermal terminal differentiation and interacts functionally with LMO4. Developmental Biology 299, 122-136.

Zhang, Y., Liu, T., Meyer, C.A., Eeckhoute, J., Johnson, D.S., Bernstein, B.E., Nusbaum, C., Myers, R.M., Brown, M., Li, W., et al. (2008). Model-based analysis of ChIP-Seq (MACS). Genome Biology 9, R137. 
Figures

Figure 1. SOX2 phosphorylation reduces clonal expansion in mouse and human SCCs

(A) Phos-tag western blots of human SCC protein lysates treated with (+) or without (-) calf intestinal phosphatase (CIP) identifies phosphorylated and un-phosphorylated SOX2.

(B) Schematic of human Flag-tagged SOX2 (F-SOX2), which is phosphorylated on Serine-37 and Serine-251. Brown bars indicate spectral coverage of tandem mass spectrometry results. Red and blue marks indicate fragment ion coverage. Phosphorylated Serine is shown in red.

(C) Immunofluorescence microscopy detects SOX2 and pS251-SOX2 in nuclei of a6-integrin positive SCC cells located along the tumor-stroma interface. DAPI stains nuclear chromatin. Scale bar $=10 \mu \mathrm{m}$.

(D) Western blots of protein lysates from human SCC cells that express endogenous SOX2 (blue arrows) along with F-SOX2 ${ }^{\mathrm{WT}}$, F-SOX2 ${ }^{\mathrm{AA}}$, or F-SOX2 ${ }^{\mathrm{DD}}$ transgenes (magenta arrows).

(E) Schematic representation of clonal competition experiment. SCC cells are infected with a pool of F-SOX2 ${ }^{A A}-p 2 A-H 2 B-G F P$ and F-SOX $2^{D D}-p 2 A-H 2 B-R F P$ containing lentiviruses at an infection rate of $\sim 5 \%$ before they are transplanted into the dermis where they expand into tumors. The ratio of F-SOX2 ${ }^{\mathrm{AA}}-\mathrm{p} 2 \mathrm{~A}-\mathrm{H} 2 \mathrm{~B}-\mathrm{GFP}$ over F-SOX2 ${ }^{\mathrm{DD}}-\mathrm{p} 2 \mathrm{~A}-\mathrm{H} 2 \mathrm{~B}-\mathrm{RFP}$ cells within a tumor indicates their relative expansion rates.

(F) Flow cytometry scatter plot depicts expansion of F-SOX2 ${ }^{A A}-p 2 A-H 2 B-G F P$ and F-SOX2 ${ }^{D D}$. p2A-H2B-RFP lineages within a representative tumor.

(G) Scatter plot indicating Log $_{2}$ of F-SOX2 ${ }^{\mathrm{AA}}-\mathrm{p} 2 \mathrm{~A}-\mathrm{H} 2 \mathrm{~B}-\mathrm{GFP}$ over F-SOX2 ${ }^{\mathrm{DD}}-\mathrm{p} 2 \mathrm{~A}-\mathrm{H} 2 \mathrm{~B}-\mathrm{RFP}$ ratio. $\mathrm{n}=6$ independent mouse and human SCC grafts. Mean $+/-$ s.e.m. $p=$ unpaired parametric two tailed Student's t-test.

(H) Fluorescence microscopy image of murine SCC section from a clonal competition experiment showing F-SOX2 ${ }^{\mathrm{AA}}$-p2A-H2B-GFP enrichment along the tumor-stroma interface while F-SOX2 ${ }^{\mathrm{DD}}$ p2A-H2B-RFP cells are detected in supra-basal and differentiated areas. a6-integrin marks tumorstroma interface. DAPI identifies nuclear chromatin. Scale bar $=50 \mu \mathrm{m}$

Figure 2. Ectopic SOX2 expression enhances self-renewal and tumor initiation in a Hras ${ }^{G 12 V}$ driven skin cancer model

(A) Hras $^{G 12 V}$ knock-in mouse embryos are infected in utero with lentiviruses that express either $S O X 2^{W T}$, SOX $2^{A A}$, or $S O X 2^{D D}$ along with CRE recombinase at e9.5.

(B) Kaplan Meier graphs showing tumor free survival times. $p<0.0001$ for all comparisons except Hras $^{G 12 V}$ vs Hras ${ }^{G 12 V} ; F-S O X 2^{D D} p=0.0075$ and $\operatorname{Hras}^{G 12 V} ; F-S O X 2^{W T}$ vs Hras ${ }^{G 12 V} ; F-S O X 2^{A A} p=$ 0.2354. $p=$ Log-rank (Mantel-Cox) test. 
(C) Tumor growth analyses of $\operatorname{Hras}^{G 12 V}(\mathrm{n}=21), \operatorname{Hras}^{G 12 V} ; F-S O X 2^{W T}(\mathrm{n}=15)$, Hras $^{G 12 V} ; F-S O X 2^{A A}$ $(n=15)$, Hras $^{G 12 V}$;F-SOX2 ${ }^{D D}(n=15)$. Mean +/- s.e.m ; $n=3$ independent experiments; all marked * at least $p<0.002, p=$ two-tailed t-test with Holm-Sidak correction.

(D) Principle component analysis (PCA) of RNA-seq data from Hras ${ }^{G 12 V}$, Hras ${ }^{G 12 V}$; F-SOX2 ${ }^{W T}$, Hras $^{G 12 V} ; F-S O X 2^{A A}$, Hras ${ }^{G 12 V} ; F-S O X 2^{D D}$ tumors. Note: One Hras ${ }^{G 12 V}$ tumor expressed endogenous Sox 2 de novo and is more closely related to $\mathrm{Hras}^{G 12 V} ; F-S O X 2^{W T}$ and Hras ${ }^{G 12 V}$; FSOX $2^{A A}$ tumors.

(E-F) Scatter plots depicting differentially expressed genes $(q<0.05)$ in Hras $^{G 12 V}$ vs. Hras ${ }^{G 12 V} ; F-$ $S O X 2^{W T}$ (E), or Hras ${ }^{G 12 V} ; F-S O X 2^{A A}$ vs Hras ${ }^{G 12 V} ; F-S O X 2^{D D}$ tumors (F). Note: the Hras ${ }^{G 12 V}$; $S O X 2^{+}$ tumor was excluded from the $\operatorname{Hras}^{G 12 V}$ data set (E). Selected differentiation and self-renewal markers are indicated in red.

(G) Representative confocal microscopy images of KLF4 stained $\operatorname{Hras}^{G 12 V} ; F-S O X 2^{A A}$ and Hras $^{G 12 V}$;F-SOX2 ${ }^{D D}$ tumors. Box-plots depicting KLF4 staining intensities in these tumors. $\mathrm{n}=6 . \mathrm{p}$ $=$ unpaired two-tailed parametric Student's t-test. Scale bar $=10 \mu \mathrm{m}$.

(H) Relative fraction of $a 6^{\mathrm{hi}} / \beta 1^{\mathrm{hi}}$ and $a 6^{\mathrm{lo}} / \beta 1^{\mathrm{lo}}$ cells in GFP-labelled Hras ${ }^{G 12 V} ; F-S O X 2^{A A}$ or Hras $^{G 12 V}$;F-SOX2 ${ }^{D D}$ SCC-lineage. Mean +/- s.e.m; $p=$ unpaired two-tailed parametric Student's ttest.

(I) Bar graph indicating self-renewal rate in $\operatorname{Hras}^{G 12 V}, \operatorname{Hras}^{G 12 V} ; F-S O X 2^{W T}, \operatorname{Hras}^{G 12 V}$;F-SOX2 ${ }^{A A}$, Hras $^{G 12 V} ; F-S O X 2^{D D}$ tumors. $\mathrm{n}>3$ tumors. Mean $+/-$ s.e.m. $\mathrm{p}=$ unpaired two tailed parametric Student's t-test.

Figure 3. SOX2 phosphorylation enhances differentiation in SCCs

(A) Sox $2^{K O}$ and SOX2-mutant replacement strategy. SOX2 function is essential in SCC cells. Therefore, we expressed F-SOX $2^{\mathrm{WT}}$, F-SOX $2^{\mathrm{AA}}$, or F-SOX2 ${ }^{\mathrm{DD}}$ transgenes before we targeted the 5' and 3' UTRs with sgRNA's to delete endogenous Sox2 with CRISPR/Cas9.

(B) SOX2 and pS251- SOX2 western blotting confirms effective Sox 2 deletion and F-SOX ${ }^{\mathrm{WT}}$, F$\mathrm{SOX} 2^{\mathrm{AA}}$, or $\mathrm{F}-\mathrm{SO} \times 2^{\mathrm{DD}}$ transgene expression.

(C) Confocal micrographs of SOX2 and pS251-SOX2 stained Sox ${ }^{K O} ; F-S O X 2^{A A}$ and Sox $2^{K O} ; F-$ SOX $2^{D D}$ SCC sections.

(D) Hematoxylin \& Eosin staining reveals a more disorganized architecture in Sox $2^{K O} ; F-S O X 2^{A A}$ compared to Sox $2^{K O} ; F-S O X 2^{D D}$ SCC sections. Scale bars $=25 \mu \mathrm{m}$.

(E) Scatter plot depicting differentially expressed genes $(q<0.05)$ between Sox $2^{K O} ; F-S O X 2^{A A}$ vs Sox $2^{K O} ; F-S O X 2^{D D}$ SCCs. Select differentiation and SCC marker genes are depicted in red. Table highlights gene sets enriched in Sox ${ }^{K O} ; F-S O X 2^{D D}$ (orange) and Sox $2^{K O} ; F-S O X 2^{A A}$ (blue) SCCs. 
(F) Confocal microscopy section reveals increased FLG2 staining in Sox $2^{K O} ; F-S O X 2^{D D}$ compared to Sox ${ }^{K O} ; F-S O X 2^{A A}$ SCCs. Box-plots illustrate differences in FLG2 staining intensities. $n=3$ tumors with 3 fields per tumor. $p=$ unpaired parametric two-tailed t-test.

(G-H) Representative scatter plots with adjunct histograms illustrating $a 6$ - and $\beta 1$-integrin staining intensities in Sox ${ }^{K O} ; F-S O X 2^{A A}$ (blue) and Sox $2^{K O} ; F-S O X 2^{D D}$ (orange) SCCs (G). Relative fraction of $a 6^{\mathrm{hi}} / \beta 1^{\mathrm{hi}}$ and $a 6^{\mathrm{lo}} / \beta 1^{\mathrm{lo}}$ cells in GFP-labelled Sox $2^{K O} ; F-S O X 2^{A A}$ and Sox $2^{K O} ; F-S O X 2^{D D}$ SCC-

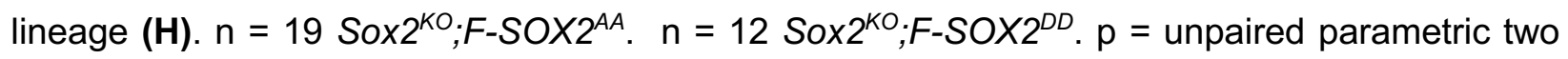
tailed Student's t-test.

(I-J) Calculated tumor initiation frequency (I) and tumor initiation rate (J) after serial transplantation of lineage marked $S O X 2^{K O} ; F-S O X 2^{A A}$ or Sox $2^{K O} ; F-S O X 2^{D D}$ cells. $p=$ Mantel-Cox Log-rank test.

\section{Figure 4. SOX2-phosphorylation affects KIf4 in a small subset of SOX2-bound sites}

(A-B) Heatmap representation of SOX2 bound gene regulatory elements that are significantly enriched (fold change > 1.5, $\mathrm{p}<0.05$ ) in $\operatorname{Hras}^{\mathrm{G12V}}$; FSOX2 ${ }^{A A}$ or $\operatorname{Hras}^{\mathrm{G12V}} ; F S O X 2^{D D}$ (A) and Sox $2^{K O} ; F-S O X 2^{A A}$ or Sox $2^{K O} ; F-S O X 2^{D D}$ (B) SCCs. Most SOX2 bound gene regulatory elements are unaffected by SOX2-phosphorylation. DREME discovered SOX-like motif and percentage of peaks containing the SOX motif. $\mathrm{n}=2$ per genotype.

(C) Intersection of differentially bound and expressed genes in $\mathrm{Hras}^{G 12 V}$;FSOX $2^{D D}$ and Sox $2^{K O}$; $F$ SOX $2^{D D}$ or Hras ${ }^{G 12 V} ; F S O X 2^{A A}$ and Sox $2^{K O} ; F-S O X 2^{A A}$ SCCs. Genes consistently changing in both, $\operatorname{Hras}^{G 12 V}$ and Sox $2^{K O}$, models are shown. $\mathrm{p}=$ exact hypergeometric probability

(D) Heatmap illustrates z-score transformed FPKM values of genes that are differentially bound and expressed dependent on SOX2-phosphorylation in SCCs. Genes are ranked based on their mean expression level and their up-regulation in SOX2 ${ }^{\mathrm{DD}}$ or SOX2 ${ }^{\mathrm{AA}}$ expressing tumors. KIf4 surfaced as the highest expressed, direct SOX2 target gene whose expression increases significantly in both of our SOX2 ${ }^{\mathrm{DD}}$ compared to SOX2 ${ }^{\mathrm{AA}}$ expressing SCC models.

(E-F) Confocal microscopy reveals significantly increased KLF4 staining in Sox $2^{K O} ; F-S O X 2^{D D}$ compared to Sox $2^{K O} ; F-S O X 2^{A A}$ tumor sections (E) and box-plots illustrate the differences in KLF4 staining densities. $n=3$ tumors and 2 fields/tumor. $p=$ unpaired parametric two tailed t-test.

Figure 5. Phosphorylated SOX2 is evicted from a KIf4 enhancer to restore KIf4 expression (A) 4C-seq and SOX2, KLF4, CTCF, MED12, and K27Ac ChIP-seq tracks identify KIf4 enhancers. $4 \mathrm{C}$-ker visualizes significant interactions between a bait at the Klf4 promoter and cis-regulatory elements by $4 \mathrm{C}$-seq (pink highlights). ChIP-seq signals - significantly $(p<0.05)$ enriched in F- 
SOX2 $2^{\mathrm{AA}}$ (blue triangle) or F-SOX2 ${ }^{\mathrm{DD}}$ (orange triangle) - identify putative SOX2-phosphorylation regulated enhancers. Orange asterisk marks site with differential KLF4 enrichment $(p<0.05)$ in $a 6^{\text {hi }} / \beta 1^{\text {hi }}$ SCC cells between SOX $2^{K O} ; F-S O X 2^{D D}$ and SOX $2^{K O} ; F-S O X 2^{A A}$ SCCs.

(B) Model illustrating KIf4 repression upon CRISPRi targeting of KIf4 ${ }^{E C 944}$.

(C) KIf4 mRNA fold change after CRISPR-interference (CRISPR-i), targeting either KIf4 ${ }^{E C 944}$ or $K I f 4^{E C 100}$. Mean +/- s.e.m. $n=4 . p=$ unpaired parametric two-tailed Student's t-test.

(D) Schematic of EC944 reporter construct. H2B-RFP is constitutively expressed under an EF1a promoter while GFP expression is controlled by KIf4 ${ }^{E C 944}$.

(E) Representative contour plot of reporter-containing SCCs separating into GFP low (orange), medium (blue), and high (red) populations (left) and scatter plot with adjunct histograms illustrating the inversely proportional expression of KIf4 ${ }^{E C 944}$ reporter and self-renewal marker a6integrin (right).

(F) Western blotting of protein lysates from sorted reporter ${ }^{+}$SCCs show SOX2 levels decrease with GFP expression while KLF4 increases.

(G) Fluorescence microscopy image showing KIf4 ${ }^{E C 944}$-GFP reporter expression. Increased GFP expression is detected in more differentiated suprabasal SCC cells. Scale bars $=10 \mu \mathrm{m}$.

(H) Tumor initiation rates from serial transplantation of GFP ${ }^{L o w} / R_{F P}{ }^{H i}$ or GFP ${ }^{H i} / R_{F P}{ }^{H i}$ cells we identified with the KIf4 ${ }^{E C 944}$-GFP reporter. $p=$ Mantel-Cox Log-rank test.

\section{Figure 6. Increased KLF4 expression drives differentiation in F-SOX2 ${ }^{\mathrm{DD}}$ SCCs}

(A) Heatmaps, histograms and violin plots of KLF4-like motif containing KLF4 ChIP-seq peaks of FACS-isolated $a 6^{\text {hi }} / \beta 1^{\text {hi }}$ and $a 6^{\text {lo }} / \beta 1^{\text {lo }}$ SCC cells reveal increased KLF4 occupancy in Sox $2^{K O} ; F$ $S O X 2^{D D}$ compared to Sox $2^{K O} ; F-S O X 2^{A A}$ tumors. DREME identified transcription factor motif at the peak summit with corresponding E-value. $p=$ unpaired non-parametric Mann-Whitney t-test.

(B) Heatmaps and violin plots (median values listed) of KLF4 ChIP-seq peaks with differential enrichment in $a 6^{\text {hi }} / \beta 1^{\text {hi }} S C C$ cells of Sox $2^{K O} ; F-S O X 2^{A A}$ or Sox $2^{K O} ; F-S O X 2^{D D}$ tumors. $n=290$ peaks $\left(\mathrm{SOX} 2^{\mathrm{AA}}\right)$ and $\mathrm{n}=317$ peaks $\left(\mathrm{SOX} 2^{\mathrm{DD}}\right) . \mathrm{p}=$ Mann-Whitney t-test.

(C) Intersection of genes associated with KLF4 bound sites that were significantly enriched in $S O X 2^{K O} ; F-S O X 2^{A A}$ or SoX $2^{K O} ; F-S O X 2^{D D}$ SCCs with differentially expressed transcripts from these tumors. $p=$ exact hypergeometric probability.

(D) GO analysis of the genes with differential KLF4 ChIP-seq enrichment and increased expression in Sox $2^{K O} ; F-S O X 2^{D D}$ tumors.

(E) Heat map representation of differentially expressed transcripts and their corresponding GO categories. 
(F) ChIP-seq tracks show increased KLF4 occupancy at the KLF4-motif (red arrowhead) containing Sox2 enhancer site that inhibits Sox2 expression.

(G) Working model: SOX2 inhibits KIf4 to maintain TPC self-renewal in basal SCC cells. SOX2 phosphorylation evicts SOX2 from a bi-stable Klf4 enhancer switch allowing residual KLF4 to bind and boost its own expression. Increased KLF4 levels promote squamous differentiation.

\section{Supplementary Figures}

\section{Figure S1.}

(A) Work flow of experimental approach to identify F-SOX2 phosphorylation sites with tandem mass spectrometry (MS/MS).

(B) Silver stained gel showing 5\% of F-SOX2 immuno-precipitate used for MS/MS.

(C) Base peak chromatogram for F-SOX2

(D-E) Spectral traces of peptides identifying phosphorylated Serine-37 (D) and Serine-251 (E).

(F) Confocal micrograph of patient SCC stained with pSer ${ }^{251}$-SOX2 and SOX2.

(G) Phos-TAG western blot of human SCC protein lysates transduced with $F$-SOX $2^{W T}$ or $F$ $S O X 2^{A A}$ transgenes showing F-SOX2 ${ }^{\mathrm{AA}}$ is, in contrast to F-SOX2 ${ }^{\mathrm{WT}}$, not phosphorylated. Black arrows mark F-SOX2 bands. Grey arrows mark non-specific bands that are also detected in untransduced cells.

(H) FACS-gating strategy to determine GFP:RFP ratio in clonal competition experiments.

Figure S2.

(A) FACS-gating strategy to isolate epidermal progenitor cells (Sca1+) and hair follicle stem cells (CD34+) from mouse back skins.

(B) Western blot of epidermal progenitor cells transduced with $\operatorname{Hras}^{G 12 \mathrm{~V}}, \mathrm{Hras}^{\mathrm{G} 12 \mathrm{~V}}$; F-SOX2 ${ }^{W T}$, $\operatorname{Hras}^{G 12 V} ; F-S O X 2^{A A}$, or Hras ${ }^{G 12 V} ; F-S O X 2^{D D}$ showing transgene but no endogenous SOX2 expression.

(C) FACS-gating strategy to sort out lineage positive cells (green, GFP+ gate) for RNAsequencing. Gating strategy for determining relative abundance of $a 6^{\mathrm{hi}} / \beta 1^{\mathrm{hi}}$ and $a 6^{\mathrm{lo}} / \beta 1^{\mathrm{lo}}$ fractions are also shown. Gates are defined based on a6- and $\beta 1$-integrin expression values of the GFPnegative, normal skin epithelial cell lineage, which serves as an internal control to define top and bottom $25 \%$ gate limits.

(D) Sox2 FPKM values in $\mathrm{Hras}^{G 12 V}$, Hras ${ }^{G 12 V} ; \mathrm{F}-S O X 2^{W T}$, Hras ${ }^{G 12 V} ; F-S O X 2^{A A}$, or Hras ${ }^{G 12 V} ; F-$ $S O X 2^{D D}$ SCCs. Red dot denotes Hras ${ }^{G 12 V}$ tumor with de novo expression of endogenous Sox2. 
Mean +/- s.e.m. n=3. Note: FPKM values for $\operatorname{Hras}^{G 12 V_{;}} ; \mathrm{F}-S O X 2^{W T}$, Hras ${ }^{G 12 V} ; F-S O X 2^{A A}$, or Hras $^{G 12 V} ; F-S O X 2^{D D}$ tumors are for the transgene and not endogenous Sox2.

(E) Confocal micrographs of tumor sections stained with pS251-SOX2 and SOX2 antibodies.

(F) Schematic of BrdU/EdU double label incorporation self-renewal assay. Mice are injected with EdU (Oh) and BrdU (2h) before sacrificing at $8 \mathrm{~h}$ after initial EdU injection. $>95 \%$ of EdU+ only cells complete one round of cell division in the $6 \mathrm{~h}$ between BrdU injection and sacrifice. Tracking EdU+ only cells (differentiated, K10+ or self-renewing, K10-) allows calculation of self-renewal rates.

\section{Figure S3.}

(A) Bar graphs showing percentages of recovered $S O X 2^{K O} ; F-S O X 2^{W T}, S O X 2^{K O} ; F-S O X 2^{A A}$, and SOX $2^{K O} ; F-S O X 2^{D D}$ clones.

(B) FACS-gating strategy to determine relative a6-integrin expression. Gates are kept constant for all samples.

\section{Figure S4.}

(A) CRISPRi gRNA targeting schematic. Independent $g R N A$ 's were designed to target either SOX2 or KLF4 motifs within the ChIP-seq peak for the F-SOX2AA (top) or F-SOX2 ${ }^{D D}$ (bottom) bound peaks in EC944 and EC1001, respectively.

(B) FACS-gating strategy to isolate $a 6^{\mathrm{hi}} / \beta 1^{\mathrm{hi}}$ and $a 6^{\mathrm{lo}} / \beta 1^{\mathrm{lo}}$ SCC cells from SOX $2^{K O} ; F-S O X 2^{A A}$, and SOX $2^{K O} ; F-S O X 2^{D D}$ tumors for KLF4 ChIP-seq experiments.

(C) Stacked bar graphs illustrate percentage of peaks with KLF4 like transcription factor motifs we discovered de-novo with DREME and their distribution in respect to the closest transcriptional start site. 


\section{Materials and methods}

\section{Resource Availability}

Further information and requests for resources and reagents should be directed to and will be fulfilled by the Lead Contact, Markus Schober (markus.schober@nyulangone.org).

\section{Materials Availability}

Plasmids generated in this study are available upon request.

\section{Data and Software Availability}

This study did not generate any new code. Data sets have been uploaded to NCBI GEO with the accession number GSE158207.

\section{Experimental Model and Subject Details}

\section{Cell lines}

Primary murine SCC-TPC lines were established from malignant tumors induced with DMBA following the previously described complete carcinogenesis protocol (Guasch et al., 2007; Schober and Fuchs, 2011; Siegle et al., 2014). Human A431 cells were grown in DMEM supplemented with $10 \%$ fetal bovine serum (FBS). Human SCC25 cells were grown in P-media (DMEM-F12 3:1 media (US Biologicals), sodium bicarbonate (Sigma), L-glutamine (Invitrogen) and Pen/Strep solution (Invitrogen)) supplemented with 10\% FBS. Differentiation was induced in primary cells by increasing $\left[\mathrm{Ca}^{+2}\right]$ from $0.05 \mathrm{mM}$ to $1.5 \mathrm{mM}$ for the times indicated.

\section{Mice}

6 week old female nude (NU/NU [088] Charles River) mice were used for all orthotopic allo/xenograft studies. Hras ${ }^{G 12 V / G 12 V}$ (Chen et al., 2009) and Rosa26 ${ }^{\text {YFP/YFP }}$ Cre-reporter mice (Jackson Laboratories) on C57BI/6 background were used for in utero transduction experiments and equal numbers of male and female mice have been analyzed in tumor formation studies. Tumors were detected by palpation, measured with digital calipers (in $\mathrm{mm}$ ), and volume calculated with the equation: $V=\frac{\pi}{6} \times l \times w^{2}$. All animal experiments were performed in 
accordance with the guidelines and approval by the Institutional Animal Care and Use Committee at New York University Langone Health.

\section{Method Details}

\section{Constructs}

Human SOX2 was PCR amplified from genomic DNA we isolated from human A431 cells using primers with the first half of the p2A tag sequence in the 3' primer. eGFP was amplified from LZRS-IRES-eGFP (Addgene 21961) using primers with the second half of the p2A tag sequence in the 5' primer. Both PCR products were purified and used as the template for a second round of PCR amplification to generate the SOX2-p2A-eGFP sequence, which was first cloned into the Zero Blunt TOPO cloning vector (ThermoFisher 450245), and then subcloned into a pcDNA 3.1 vector containing the flag-flag-streptavidin-streptavidin (FFSS) tag (generous gift from Dr. Michele Pagano) using the EcoRV restriction site. The full FFSS-SOX2-p2A-eGFP (FSOX2) sequence was excised from the pcDNA3.1 vector with the restriction enzymes Nhel and EcoRV and ligated into pLKO.1 (Addgene 10878). FSOX2 ${ }^{\mathrm{AA}}$ and FSOX2 ${ }^{\mathrm{DD}}$ mutant constructs were obtained by sequential site directed mutagenesis and sequenced to ensure no other mutations were present.

The p2A-H2B-GFP sequence was amplified from pLKO Histone H2B-GFP (Addgene 25999) and the p2A-H2B-RFP sequence was amplified from pLKO Histone H2B-mRFP1 (Addgene 26001 ) with primers containing the $\mathrm{p} 2 \mathrm{~A}$ sequence at the 5 ' primer. Subsequent steps to generate FFSS-SOX2-p2A-H2B-eGFP or - FFSS-SOX2-p2A-H2B-mRFP constructs were the same as described above.

For in utero transduction experiments, human SOX2 from the CCSB Broad ORF library (Yang et al., 2011) was cloned into the pLEX_307 (Addgene 41392) vector. SOX $2^{\mathrm{AA}}$ or SOX2 $2^{\mathrm{DD}}$ constructs were generated by site directed mutagenesis with PCR using the human SOX2 ${ }^{\mathrm{WT}}$ plasmid as template.

pLKO-HRas ${ }^{G 12 V}$-Puro was generated by subcloning HRas ${ }^{G 12 V}$ from PQCXIX-PGK-Hras ${ }^{V 12}$. IRES-H2B-RFP (generous gift from Dr. Paul Khavari) into the pLKO vector.

To make the guide RNA backbone, the puromycin resistance gene was PCR amplified from the pLKO.1 vector and cloned into the pEHL958-pLKO-GFP-sgRNA backbone (generous gift from Dr. Eva Hernando-Monge), by excising GFP with BamHI and Sall and replacing it with the puromycin resistance gene. Individual guide RNAs were designed with the Benchling CRISPR gRNA tool and cloned into this vector using the Bbsl restriction site. 
The EC944-eGFP reporter was constructed by PCR amplification of mouse genomic DNA with a primer pair that introduced $\mathrm{Kpnl}$ and $\mathrm{BsaBI}$ restrictions sites and it was cloned into corresponding sites of a lentiviral Notch-reporter (kind gift from Scott Williams (Williams et al., 2011)).

\section{Cell culture}

Primary SCC cells were infected with VSV-G psuedotyped lentiviruses. Lentiviruses were produced by transfecting 293FT cells with pLKO sgRNA or FSOX2 expression vectors and helper plasmids pMD2-VSVg and pPAX2 (Addgene plasmid 12259 and 12260) using Lipofectamine 2000 (Thermo Fisher). 293FT cells were cultured in DMEM (Gibco) media supplemented with $10 \%$ FBS and Pen/Strep solution. Virus containing media was harvested $48 \mathrm{~h}$ and $72 \mathrm{~h}$ posttransfection and filtered with $0.45 \mu \mathrm{m}$ pore size mixed cellulose esters syringe filters (EMD Millipore SLHA033SS). For lentiviral infection, $3 \times 10^{4}$ cells per well were plated into a 6 well plate, incubated with a 1:3 dilution of viral supernatant with $30 \mathrm{ug} / \mathrm{mL}$ of polybrene and spun at $1,100 \mathrm{x}$ $\mathrm{g}$ for $30 \mathrm{~min}$ at $37^{\circ} \mathrm{C}$. For FSOX2 stable cell lines, eGFP+ cells were selected by FACS using a BD FACSAria II equipped with 405, 488, 561, 647, and 355nm lasers. Primary epidermal progenitor cells (CD49f+/Sca1+/ CD34-) were extracted from 21 day old mice with trypsin, isolated by FACS and cultures were established on 3T3 feeder layers. Cells were transduced with pLKOHRas $^{\mathrm{G} 12 \mathrm{~V}}$-Puro on 3 T3 feeder layers. Transduced cells were selected with $1 \mu \mathrm{g} / \mathrm{mL}$ puromycin (Sigma-Aldrich) containing media for 2 days before they were transduced with pLKO-FSOX2p2A-eGFP, pLKO-FSOX2 ${ }^{A A}-p 2 A-e G F P$, or pLKO-FSOX2 ${ }^{D D}-p 2 A-e G F P$ and sorted for eGFP expression.

\section{CRISPR/Cas9 knockouts}

For SOX $2^{\mathrm{KO}}$ - SOX2 replacement experiments, primary SCC cultures were first transduced with pLKO-FSOX2 ${ }^{\mathrm{AA}}-\mathrm{p} 2 \mathrm{~A}-\mathrm{eGFP}$ or pLKO-FSOX2 ${ }^{\mathrm{DD}}$-p2A-eGFP before the entire Sox2 coding region was deleted using two gRNAs targeting the 5' and 3' UTR's. Guide RNA's were chosen based on similar cutting efficiency scores and to minimize off targets using the Benchling CRISPR gRNA design tool. Cells were infected with sgRNAs, selected with $1 \mathrm{ug} / \mathrm{mL}$ puromycin (SigmaAldrich) containing media for 2 days before they were infected with lenti-Cas9-Blast (Addgene 52962) and selected with $5 \mathrm{ug} / \mathrm{mL}$ blasticidin for 3 days. After selection, 100 cells were plated in a $10 \mathrm{~cm}$ dish and single clones were picked with $6 \mathrm{~mm}$ cloning cylinders when clone sizes reached $\sim 100$ cells. Clones were screened by PCR amplification of the Sox 2 locus with primers flanking 
the Sox2 coding sequence using gDNA as template as well as western blotting, where endogenous SOX2 and FSOX2 can be distinguished based on the FFSS-tag size difference.

\section{CRISPRi}

Independent gRNAs targeting either the SOX2 or KLF motif within peaks EC944 or EC1001 were designed using the Benchling gRNA design tool (www.benchling.com). TPC cell lines were infected with rTTa-N144 (Addgene \#66810) and selected with hygromycin (10ug/mL). The cells were then infected with TRE-dCas9-KRAB-IRES-GFP (Addgene \#85556), induced with doxycycline $(1 \mu \mathrm{g} / \mathrm{mL})$ for $48 \mathrm{~h}$, and FACS sorted for GFP+ cells. The GFP ${ }^{+}$population was expanded and lentivirally infected with the gRNAs before selection with puromycin $(2 \mathrm{ug} / \mathrm{mL})$. dCas9-KRAB expression was induced with doxycycline $(1 \mu \mathrm{L} / \mathrm{mL})$ for $48 \mathrm{~h}$ before harvesting cells for protein and RNA to check for changes in Klf4 expression. KRAB-dCas9-HA expression was confirmed by western blotting using anti-HA antibodies.

\section{Clonal competition}

Lentiviruses containing FSOX2-p2A-H2B-eGFP or FSOX2-p2A-H2B-mRFP were generated by mixing equimolar concentrations of both plasmid DNAs during transfection of 293FTs. Human A431 or primary murine TPCs were infected at low MOI. The percentage of GFP:RFP containing cells were checked on a BD LSRII UV flow cytometer equipped with 405, 488, 561, 647, and $355 \mathrm{~nm}$ lasers $72 \mathrm{~h}$ after infection. $1 \times 10^{5}$ cells from pools that contained $\sim 5 \% \mathrm{GFP}+\mathrm{RFP}+$ cells were used for each transplantation.

\section{Tumor isolation and flow cytometry}

Tumors from murine allografts were isolated as previously described (Sastre-Perona et al., 2019). Briefly, tumor tissue was separated from normal skin, blood vessels and connective tissue as much as possible before mincing and incubation in HBSS (Gibco) with $1.25 \%$ Collagenase Type IA (Sigma) for $40 \mathrm{~min}$ at $37^{\circ} \mathrm{C}$ and shaking in an orbital shaker at $60 \mathrm{rpm} .62 .5 \mathrm{U} / \mathrm{mL}$ DNAsel (Worthington) is added afterwards and incubated for another $10 \mathrm{~min}$, shaking. The tumor suspensions are filtered through $70 \mu \mathrm{m}$ then $40 \mu \mathrm{m}$ mesh strainers (Thermo Fisher). Remaining undigested tumor pieces are incubated with 0.25\% trypsin (Gibco) for 10min, 60rpm before filtering through the mesh strainers again. Cell suspensions are spun down at $300 \times \mathrm{g}$ for $10 \mathrm{~min}$ at $4^{\circ} \mathrm{C}$. The supernatant is discarded and the tumor pellet is washed with $1 \times$ RBC lysis buffer (Biolegend), pelleted at $300 \times \mathrm{g}$ for $3 \mathrm{~min}$, and washed with staining buffer ( $2 \%$ chelexed FBS and $0.5 \mathrm{U} / \mathrm{mL}$ DNAsel in dPBS). 
For flow cytometry, tumor suspensions were stained in the dark for $30 \mathrm{~min}$ on ice and washed after staining to remove excess antibodies. DAPI (40,6-diamidino-2-phenylindole; D1306, Invitrogen) was used for live/dead cell exclusion. FACS data was recorded on either the BD FACSAria II if sorting was required or the BD LSRIIUV if no sorting was required. Flow cytometry data was analyzed using FlowJo software.

\section{Serial transplantations}

For limited dilution serial transplantation studies, parental tumors were processed as described above and sorted on a BD FACSAria II flow cytometer. Total GFP lineage positive cells were sorted out, serially diluted to obtain 1,000 or 100 cells, mixed with $50 \%$ Matrigel solutions, and intradermally injected into nude recipient mice (Sastre-Perona et al., 2019).

\section{Immunoprecipitation and tandem mass spectrometry}

Immunoprecipitation (IP): Human SCC25 cells expressing FSOX2 ${ }^{\mathrm{WT}}$ were lysed in IP buffer (20mM HEPES pH8.0, 10mM KCl, 0.15mM EDTA, 0.15mM EGTA, 100mM NaCl, 1\% NP-40), sheared with an insulin syringe to break the nuclei, and incubated for 30 minutes at $4^{\circ} \mathrm{C}$ while rotating. Lysates are pelleted at max speed in a table top centrifuge ( 21000rpm) for $10 \mathrm{~min}, 4^{\circ}$, and the supernatant is combined. Lysate pre-clearing was done with $50 \mu \mathrm{L}$ of IP buffer washed protein $\mathrm{A}$ agarose beads (Cell Signaling) for $1 \mathrm{~h}$ at $4^{\circ} \mathrm{C}$, rotating. Protein $\mathrm{A}$ beads were pelleted at $4000 \mathrm{rcf}$ for $4 \mathrm{~min}$ at $4^{\circ} \mathrm{C}$ and the supernatant was collected. 50 $\mu \mathrm{L}$ Anti-Flag M2 beads (Sigma) were washed twice with IP buffer, added to the supernatant, and incubated overnight while rotating at $4^{\circ} \mathrm{C}$. Flag beads were washed $3 \mathrm{x}$ with IP buffer, $1 \mathrm{x}$ with low salt, $1 \mathrm{x}$ with high salt, and $2 x$ with $\mathrm{LiCl}$ salt wash buffers $(20 \mathrm{mM}$ Tris-HCl pH8.0, 2mM EDTA, 0.1\% SDS, 1\% Triton X100 and $167 \mathrm{mM} \mathrm{NaCl}$ or $500 \mathrm{mM} \mathrm{NaCl}$ (low/high salt washes), and 10mM Tris-HCl pH8.0, 1mM EDTA, $1 \% \mathrm{NP}-40,1 \%$ deoxycholic acid, and $250 \mathrm{mM} \mathrm{LiCl}$ for $\mathrm{LiCl}$ salt wash), changing to a new tube every wash to reduce background. $5 \%$ of the Flag beads were used to check the concentration and IP efficiency by silver stain before using the rest for mass spectrometry.

Mass spectrometry: IP'ed FSOX2 ${ }^{\mathrm{WT}}$ on Flag beads was reduced with DTT at $57^{\circ} \mathrm{C}$ for $1 \mathrm{~h}$, alkylated with iodoacetic acid at RT in the dark for 45min, and run on NuPAGE $4-12 \%$ bis-tris gels (Life Technologies). The gel was stained with GelCode Blue (Thermo Fisher) and the FSOX2 band was excised based on size. Excised gel pieces were destained in a 1:1 v/v solution of methanol and $100 \mathrm{mM}$ ammonium bicarbonate, dried, and digested with $\mathrm{CNBr}$ in $70 \% \mathrm{TFA}$ overnight in the dark at RT. Digestion was quenched with $1 \mathrm{~mL}$ of LCMS water and dried in a SpeedVac before being further digested by 250 ng of sequencing grade modified trypsin 
(Promega). $300 \mu \mathrm{L}$ of $100 \mathrm{mM}$ ammonium bicarbonate and $200 \mu \mathrm{L}$ of $1 \mathrm{M}$ ammonium bicarbonate was added to the gel pieces and digested overnight. $500 \mu \mathrm{L}$ of R2 $20 \mu \mathrm{m}$ Poros beads (Life technologies) in $5 \%$ formic acid and $0.2 \%$ TFA was added to the sample and shaken for $4 \mathrm{~h}$ at $4^{\circ} \mathrm{C}$. The beads were loaded onto equilibrated C18 ziptips (EMD Millipore) using a microcentrifuge for $30 \mathrm{sec}$ at $6000 \mathrm{rpm}$. Gel pieces were rinsed $3 \mathrm{x}$ with $0.1 \%$ TFA and also loaded onto ziptips. Extracted poros beads were washed with $0.5 \%$ acetic acid and peptides eluted by the addition of $40 \%$ acetonitrile (ACN) in $0.5 \%$ acetic acid followed by $80 \%$ CAN in $0.5 \%$ acetic acid. The organic solvent was removed using a SpeedVac and the protein reconstituted in $0.5 \%$ acetic acid. 1/3 of the sample was analyzed through MS. Peptides were separated using an online LCMS using the autosampler of an EASY-nLC 1000 (Thermo Scientific) and gradient eluted from the column directly to the Orbitrap Elite MS (Thermo Scientific) using a $1 \mathrm{hr}$ gradient. High resolution full MS spectra were acquired with a resolution of 60,000 , an AGC target of $1 \times 10^{6}$, with a maximum ion time of $200 \mathrm{~ms}$, and a scan range of $300-1500 \mathrm{~m} / \mathrm{z}$. Following each full MS, fifteen data-dependent high resolution HCD MS/MS spectra were acquired. All MS/MS spectra were collected using the following instrument parameters: resolution of 15,000, AGC target of $5 \times 10^{4}$, max ion time of $100 \mathrm{~ms}$, one microscan, $2 \mathrm{~m} / \mathrm{z}$ isolation window, fixed first mass of $150 \mathrm{~m} / \mathrm{z}$, and NCE of 30 . MS/MS spectra were searched against the SOX2 sequence using Byonic for phosphorylation modifications.

\section{In utero injections}

Lentiviral transduction in vivo via ultrasound-guided in utero microinjection was performed as previously reported (Beronja et al., 2010; Ying et al., 2018). Briefly, developmental stage of mouse embryos was determined by Vevo 1100 animal ultrasound imager using MS550D transducer (Visualsonics). $1 \mu \mathrm{l}$ of virus diluted to achieve desired transduction level was injected into amniotic cavity of E9.5 day embryos using a glass capillary needle fitted on Celltram Vario micro injector (Eppendorf).

\section{Self-renewal assay}

We used the previously described assay (Ying et al., 2018) as follows: We first administered EdU to animals, followed by BrdU injection 2 hours later. Six hours later we harvested epidermis and detected EdU, BrdU signal as well as differentiation marker K10 by immuno-fluorescence microscopy. We defined the number of EdU+ only cells as E. We calculated the rate of progenitor cell renewal of the EdU+ only cells $(E)$ using the following equation: rate of renewal = (number of K10- E cells) / (total number of E cells). 


\section{Western blotting}

Protein lysates were prepared in RIPA buffer $(150 \mathrm{mM}$ sodium chloride, $0.1 \%$ Triton-X 100, $0.5 \%$ SDS and $50 \mathrm{mM}$ Tris $\mathrm{pH} 8$ in ddH2O) supplemented with Mini EDTA-free protease inhibitor tablets (04693159001, Roche). Protein concentrations were determined with the Pierce BCA Protein Assay Kit (23225, Pierce). Lysates were boiled with 5x Laemmli buffer (6\% SDS, 10\% $\beta$ mercaptoethanol, $30 \%$ glycerol, $0.006 \%$ bromophenol blue, $0.188 \mathrm{M}$ Tris- $\mathrm{HCl}$ ) for $10 \mathrm{~min}$ at $95^{\circ} \mathrm{C}$. Novex Sharp Pre-stained Protein Standard (LC5800 Thermo Fisher) was used for molecular weight markers. $30 \mu \mathrm{g}$ of protein was loaded per lane. Gel electrophoresis was performed using a $10 \%$ bis-tris gel run at $120 \mathrm{~V}$ for $2.5 \mathrm{~h}$. Gels were transferred for $1 \mathrm{~h}$ at $4{ }^{\circ} \mathrm{C}$ at $100 \mathrm{~V}$ to a $0.45 \mu \mathrm{m}$ nitrocellulose membrane (GE Healthcare Amersham). Transfer efficiency was determined with Ponceau S staining (0.1\% (w/v) Ponceau S in 5\% (v/v) acetic acid) and membranes blocked with blocking buffer (5\% non-fat dry milk in TBST). Membranes were rinsed with TBST before incubating with primary antibodies in blocking buffer overnight. Membranes were washed 3x with TBST, incubated with HRP-conjugated secondary antibodies in blocking buffer for $1 \mathrm{~h}$ at RT and washed $3 x$ with TBST. Membranes were developed using Supersignal West Pico or Femto Chemiluminscent substrate (Life Technologies, \#34080 add \#30095) and exposed to X-ray film (F-9024-8_10, GeneMate) using a Kodak X-Omat 2000A Processor.

Antibodies used for western blotting were SOX2 (1:1,000; Abcam, ab92494), pSOX2 (1:1000; Cell Signaling, 92186), FLAG (1:2000, Sigma, F3165), KLF4 (1:1,000; R\&D, AF3158), GFP (1:2000, Abcam, Ab290), Vinculin (1:10000; Sigma, V9131), HRP donkey anti-rabbit lgG $(H+L)$ (1:3000; Jackson, 711-035-152), HRP donkey anti-mouse IgG $(H+L)$ (1:3000; Jackson, 711-035151), and HRP donkey anti-goat IgG $(H+L)$ (1:3000; Jackson, 705-035-147).

\section{Immunofluorescence, histology, and imaging}

Unfixed tumors were embedded in OCT (Sakura Finetek \#4483) and frozen. Sections were cut on a Leica cryostat to a thickness of $10 \mu \mathrm{m}$ and mounted on SuperFrost Plus (Thermo Fisher) slides. Slides were air-dried for $10 \mathrm{~min}$, fixed for $10 \mathrm{~min}$ with $4 \%$ formaldehyde, rinsed with PBS, permeabilized and incubated in blocking buffer ( $5 \%$ normal donkey serum, $1 \%$ BSA, $0.3 \%$ Triton $\mathrm{X}-100$ in PBS) for $1 \mathrm{~h}$ at RT, and stained with primary antibody diluted in blocking buffer at $4^{\circ} \mathrm{C}$ overnight in a humidity chamber. Slides were washed 3x with PBS the next day, incubated with fluorophore conjugated secondary antibodies and Hoechst 33342 (83218, Anaspec) for 1h at RT, washed $3 x$ with PBS, and mounted with ProLong Gold (Thermo Fisher) reagent. Imaging was 
performed on a Nikon Eclipse TiE microscope or a Zeiss LSM780 confocal microscope. Images were analyzed in ImageJ (Fiji).

For H\&E stains, tissue slides were fixed as above, washed 2x with $1 x$ PBS, $1 x$ with $\mathrm{H} 2 \mathrm{O}$, stained in hematoxylin (Thermo Fisher \#7231) for 3min, rinsed with $\mathrm{H}_{2} \mathrm{O}$, and counter-stained with eosin (Thermo Fisher \#71204) for between 5-10min. Slides were then rinsed with $\mathrm{H}_{2} \mathrm{O}$, followed by consecutive washes for $1 \mathrm{~min}$ each of $90 \%, 100 \%$, and $100 \% \mathrm{EtOH}$ before dehydration $2 \mathrm{x}$ with xylenes at 3 min each and mounting.

Primary antibodies used in immunofluorescence (IF): CD49f (1:200; Biolegend, 313618), SOX2 (1:1000; Abcam, Ab92494), SOX2 (1:500, R\&D, AF2018), pSOX2 (1:500; Cell Signaling Technologies, 92186), KLF4 (1:1000; R\&D, AF3158), Filaggrin (1:1000; BioLegend, 905801), BrdU MoBU-1 (1:100; Thermofisher, B35128) and K10 (1:1000; Biolegend, Poly19054). Secondary antibodies used in IF: AlexaFluor 488 Donkey a-goat IgG (Thermo Fisher, A11055), AlexaFluor 488 Affinipure Donkey a-rabbit IgG (Jackson, 711-545-152), Rhodamine Red Affinipure Donkey a-rabbit IgG (Jackson, 711-295-152), AlexaFluor 568 Donkey a-goat (Thermo Fisher, A11057).

\section{Chromatin immunoprecipitation and sequencing}

For SOX2 ChIP-sequencing, $1 \times 10^{7}$ cells were fixed for 10 minutes with $1 \%$ formaldehyde in PBS while rotating at RT and quenched with $0.125 \mathrm{M}$ glycine for another 5 min while rotating at RT. After washing twice with PBS, cells were collected and chromatin prepared according to the ChIP-IT High Sensitivity (Active Motif, 53040) protocol. Briefly, cell pellets were re-suspended in $10 \mathrm{~mL}$ of chromatin preparation buffer supplemented with protease inhibitor cocktail (PIC) and PMSF, nuclei were broken with a dounce homegenizer on ice, and chromatin sheared with a Diagenode Bioruptor 300 at maximum intensity for $20 \mathrm{x}$ cycles of $30 \mathrm{sec}$ on and $30 \mathrm{sec}$ off. A small aliquot of sonicated chromatin was used to confirm sheared chromatin was between 200-1200bp in size, according to manufacturer protocol. IP was performed overnight at $4^{\circ} \mathrm{C}$ on $30 \mu \mathrm{g}$ of

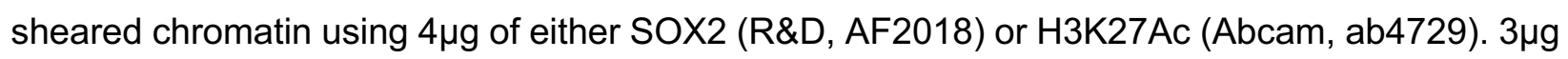
$(10 \%)$ human SCC chromatin was included in the IP as an independent spike-in normalization control. Immunoprecipitated samples were washed, de-crosslinked, and DNA purified with a spin column and eluted in $37 \mu \mathrm{L}$ of DNA Purification Elution buffer.

Libraries were prepared by concentrating eluted DNA in a SpeedVac and reconstituting in $12 \mu \mathrm{L}$ of Molecular Biology Grade Water (Corning, $46-000-\mathrm{Cl}$ ). $2 \mu \mathrm{L}$ of the solution were used to determine the DNA concentration on a Qubit 2.0 fluorometer (Thermo Fisher). Libraries were prepared using the SMARTer ThruPLEX DNA-Seq kit (Takara Bio, R400675) using barcode 
indexes from the SMARTer DNA Single Index Kits (Set A or Set B, R400695 or R400697). Library concentration and quality was assessed by Tapestation after the first round of PCR amplification and amplified for 2-3x more cycles if necessary, before AMPure XP bead (Beckman Coulter, A63880) cleanup to remove excess barcodes. Library concentration and quality was assessed a second time by Tapestation before equimolar amounts of each sample were multiplexed and run on either an Illumina HiSeq 4000 (single read 50) or a NovaSeq 6000 (SP 100bp flow cell) instrument.

\section{ChIPmentation}

ChIPmentation was performed as previously described (Schmidl et al., 2015). For KLF4 ChIPseq experiments, $1 \times 10^{6}$ basal and suprabasal SCC cells were isolated by flow cytometry as described in Figure S4C. H3K27Ac ChIP-seq experiments were conducted with $2 \times 10^{6}$ cultured TPCs. Cells were fixed, first with ChIP crosslink Gold (Diagenode C01019027) and then with formaldehyde and resuspended in $200 \mu \mathrm{L}$ of sonication buffer (10mM Tris pH8, $0.25 \%$ SDS, $2 \mathrm{mM}$ EDTA) supplemented with PIC, and sonicated as described above. Chromatin was then diluted 1:1.5 with equilibration buffer (10mM Tris, $233 \mathrm{mM} \mathrm{NaCl}, 1.66$ Triton X-100. 0.166 DOC, 1mM EDTA, PIC) and incubated over night with 4 ug of anti-KLF4 or H3K27Ac antibodies, followed with a 3-hour incubation with $10 \mu$ protein G Dynadeabs (previously blocked in $1 \% \mathrm{BSA}$ ). Immunocomplexes were washed twice with RIPA-LS, RIPA-HS, RIPA-LiCl and 10mM Tris pH8 supplemented with PIC (LS: $10 \mathrm{mM}$ Tris-HCl pH8, 140mM NaCl, 1mM pH8 EDTA, 0.1\% SDS, 0.1\% Na.Deoxycolate, 1\% Triton X-100;HS: 10mM Tris-HCL pH8, 1mM EDTA, 500mM NaCl, 1\% Triton X-100, 0.1\% SDS, 0.1\% DOC; LiCl: 10mM Tris-HCl pH8, 1mM EDTA,250mM, 0,5\% NP$40,0.5 \% \mathrm{DOC})$, before they were incubated with the tagmentation reaction, using $1 \mu \mathrm{L} \operatorname{Tn} 5$ in $1 \mathrm{x}$ Tagmentation buffer (50mM Tris $\mathrm{pH} 8,25 \mathrm{mM} \mathrm{MgCl} 2,50 \% \mathrm{v} / \mathrm{v}$ dimethylformamide) for $1 \mathrm{~min}$ at 37 C. Tagmented DNA was washed twice with RIPA-LS and TE, eluted, and crosslinks were reversed overnight in elution buffer $(10 \mathrm{mM}$ Tris- $\mathrm{HCl}$ pH8, 05mM EDTA pH8, 300mM NaCl, $0,4 \%$ SDS) with proteinase K. DNA was then purified using QIAGEN Min Elute PCR purification kit (28004) in a final volume of $10 \mu \mathrm{l}$. To reduce GC and size bias during the PCR amplification step, the number of PCR cycles were optimized by qPCR and amplification was stopped before saturation, at a cycle number corresponding to a quarter of the maximum fluorescent intensity. Libraries were cleaned and size selected using SPRI beads (AMPureXP beads, Beckman). Multiplexed libraries were sequenced on an IlluminaHiSeq 4000 Genome Analyzer using the 50base single-end read method.

\section{RNA-seq library preparation and sequencing}


Total RNA was isolated from $3 \times 10^{5}$ HRas $^{\mathrm{G} 12 \mathrm{~V}}{\text {, } \text { HRas }^{\mathrm{G} 12 \mathrm{~V}} ; \mathrm{FSOX} 2^{\mathrm{WT}} \text {, HRas }}^{\mathrm{G} 12 \mathrm{~V}} ; \mathrm{FSOX} 2^{\mathrm{AA}}$, and $\mathrm{HRas}^{\mathrm{G} 12 \mathrm{~V}} ; \mathrm{FSOX} 2^{\mathrm{DD}}$ or SOX $2^{\mathrm{KO}} ; \mathrm{FSOX} 2^{\mathrm{AA}}$ and $\mathrm{SOX} 2^{\mathrm{KO}} ; \mathrm{FSOX} 2^{\mathrm{DD}}$ GFP-lineage marked SCC cells using Qiazol (Qiagen) and Direct-zol RNA MiniPrep Kits (Zymo Research, R2052). RNA quality was assessed using an Agilent 2100 Bioanalyzer before preparing ribo-depleted, multiplexed, paired end libraries with the Illumina TruSeq Stranded Ribozero Gold kit. Multiplexed libraries were sequenced on an Illumina NovaSeq 6000 using the S1 100bp flow cell.

\section{C library preparation and sequencing}

SCC cultures were process and analyzed as described (Rocha et al., 2016; Simonis et al., 2006) with some modifications. 10 million SCC cells were fixed in formaldehyde $2 \% 10$ minutes at RT rocking. After formaldehyde neutralization with glycine $0.125 \mathrm{M}$, cells were washed with cold PBS, resuspended in lysis buffer (50mM Tris, 150mM NaCl, 5mM EDTC, 0.5\% NP-40, 1\% TX100 , with complete protein inhibitors Roche) and dounce-homogenized to extract nuclei. Nuclei were digested with Dpnll overnight (NEB R0543M), followed by an overnight ligation with T4 DNA ligase (Invitrogen 15224). Ligated DNA was reverse-crosslinked overnight with proteinase $\mathrm{K}$ and RNAse $A$ and was purified by phenol-chloroform extraction. DNA was digested overnight with the secondary enzyme Csp6I (Fermentas ER0211) and ligated overnight with T4 DNA ligase (NEB M0202). Next, DNA was phenol-chloroform isolated and column purified (GE28-9034-71). Digestion and ligation efficiencies were confirmed after each step by reverse crosslinking, phenolchloroform DNA extraction and electrophoresis in agarose gel.

Libraries were made using the Expand Long Template PCR system (Roche) with primers designed to target the KLF4 promoter. Primers included Illumina adaptor sequence and a specific barcode for each replicate. Final libraries were purified using columns (GE28-9034-71), size selected with AMPure XP beads (Beckman) and quality assessment on the TapeStation. Multiplexed libraries were sequenced with a NextSeq 500 using the High Output Kit v2.5 (150 Cycles) and paired-end settings.

Demultiplexed reads were mapped to a reduced mm10 genome containing 44bp flanking regions of Dpnll restriction sites and interactions were analyzed with 4C-ker to identify cisinteractions as described (Raviram et al., 2016).

\section{Quantification and statistical analysis}

All experiments were carried out single blinded. All in vivo quantitative data were collected from experiments performed in at least triplicate independent tumors and expressed as mean \pm s.e.m. Box plots show median, $25^{\text {th }}$ and $75^{\text {th }}$ percentile, minimum, and maximum. Differences 
between groups were tested using unpaired two-tailed Student's t-tests, Log-Rank (Mantel Cox) tests, or otherwise indicated in figure legends. Statistical analyses were performed with Graphpad Prism 8 software. Differences were considered significant if $p<0.05$ and $p$-values are shown on main and supplemental figures. For clonal competition assays, total population size was counted as percentage of $\mathrm{GFP}^{+}$or $\mathrm{RFP}^{+}$events out of the total live tumor singlets population and expressed as $a \log _{2}$ fold enrichment compared to pre-injection values. For KLF4 and FLG2 staining quantification, total signal intensity in the FLG2 channel was divided by the number of cells in each field to generate an average signal intensity per cell per field. For KLF4 basal and suprabasal quantification, basal cells were defined as cells directly adjacent to the integrin a6+ basement membrane. Suprabasal SCC were identified by excluding basal SCC cells and stromal cells. KLF4 staining intensity was then measured and divided by either the number of basal or suprabasal cells to get KLF4 staining intensity per cell per field.

Two approaches were taken for gating strategies in the a6- and $\beta 1$-integrin high vs low comparison. To normalize for sample-to-sample variation in the HRas ${ }^{\mathrm{G} 12 \mathrm{~V}}$ model, $a 6^{\mathrm{hi}} / \beta 1^{\mathrm{hi}}$ and $a 6^{\mathrm{lo}} / \beta 1^{\mathrm{lo}}$ gates in the $\mathrm{GFP}^{+}$SCC cell lineage were defined relative to $\alpha 6$ - and $\beta 1$-integrin levels of GFP- $^{-}$skin epithelial cells. For the Sox $2^{\mathrm{KO}}$ model, we ensured equal spectral compensation by matching compensation bead (BD \#552844 and \#552843) intensity within a narrow range for every run. FACS gates were defined on one tumor and applied to every other tumor in the cohort.

Statistical significance for gene set intersections was calculated using the sizes of the two gene sets, the overlap between the two gene sets, and the size of all genes in the background mouse genome, using online calculation tools (http://nemates.org/MA/progs/overlap stats.html).

\section{ChIP-seq analysis}

Two biological replicates were sequenced in each ChIP-seq experiment. Mouse SOX2 ChIP-seq and human spike-in ChIP-seq reads were aligned to the mm10 mouse genome or hg38 human genome using bowtie2, version 2.3.4.1 (Langmead and Salzberg, 2012). Aligned reads with a quality score of $<30$ were discarded with Samtools, version 1.9 (Li et al., 2009) and PCR duplicates were discarded using Picard-tools, version 1.88 (http://broadinstitute.github.io/picard/). IGV viewer files were generated using igvtools, version 2.4.1 (Robinson et al., 2011) with the following settings: -z 5 -w 25 -e 250. A scale factor was calculated from the number human spikein reads aligned to the human genome. The sample with the lowest human reads was set as 1 and the rest of the samples scaled down to match it using picard-tools downsampleSam, STRATEGY=Chained, RANDOM_SEED=8 to randomly remove reads from the .bam files with a probability equal to the scale factor. ChIP-seq peaks were called using MACS2, version 2.1.1 
(Feng et al., 2012; Zhang et al., 2008) in comparison to input controls with default settings and a -q $.01\left(\right.$ Sox $2^{\mathrm{KO}}$ ) or -q 0.001 (HRas $^{\mathrm{G} 12 \mathrm{~V}}$ ) cutoff. Motif discovery was performed using MEME-ChIP, version 5.0.2 (Bailey et al., 2009; Machanick and Bailey, 2011) on the ChIP peak summits $\pm 100 \mathrm{bp}$ with default settings. Peaks containing the SOX motif were combined to generate a "peakome" that comprises all called peaks from all samples of an experiment. Motif containing peak percentages were calculated based on the number of motif containing peaks within all peaks in the peakome. The Genomic Regions Enrichment of Annotations Tool (GREAT, (McLean et al., 2010)) was used to determine the SOX2 peak distributions (from nearest TSS) and identify nearest neighbor genes relative to SOX2 bound sites. Two replicates were sequenced for KLF4 and H3K27Ac, and one replicate was sequenced for MED12. Analyses were performed as described for SOX2 ChIP-seq with some modifications. MACS was used with a $-q$ q 0.05 cutoff for KLF4 ChIP-seq studies, and the -broad option with the default q cutoff was used for H3K27Ac ChIP-seq studies. No spike-in normalization controls were used in these experiments.

To compare overall ChIP-seq signals and distributions, Deeptools, version.3.2.1 (Ramírez et al., 2014) was used to create a .bw file from the .bam files (--exactScaling, RPGC normalization, and bin size 10 settings), quantifying the signal using computeMatrix (reference-point mode, -b 500 and -a 1500 settings). Heatmaps, profiles (histograms), and distributions were generated from the matrix file.

RNA-seq and ChIP-seq data were integrated by extracting the closest up and downstream genes associated with each peak using GREAT and overlapping gene lists. Gene ontology analyses were performed using AmiGO, version 2.5.12 (Carbon et al., 2009).

For statistical analysis, reads within all peaks in the peakome genomic regions were counted from down-sampled .bam files (SOX2) or original .bam files (KLF4) using HTSeq (Anders et al., 2015) and differentially bound regions were determined using DESeq2 (Love et al., 2014) with a $p<0.1$ (KLF4 ChIP-seqs), $p<0.05$ and $\log _{2}$ fold change cutoff of 0.585 (1.5 fold change) (HRas ${ }^{\mathrm{G} 12 \mathrm{~V}}$; SOX2 and SOX $2^{\mathrm{KO}}$; SOX2 ChIP-seqs). Regions with low coverage ( $<10$ read counts) were discarded.

\section{RNA-seq analysis}

Three replicates were sequenced for each RNA-seq. RNA-seq data were analyzed using the Tuxedo Suite of tools (Kim et al., 2013; Langmead and Salzberg, 2012; Trapnell et al., 2012) RNA-seq reads were aligned to the mouse mm10 genome using the tophat/bowtie2 aligner (tophat v2.1.1, bowtie v2.3.4.1) and -no-coverage-search option. Transcriptome quantification and differential expression (DE) analysis was performed using the 
Cufflinks/Cuffquant/Cuffdiff/CummeRbund pipeline (cufflinks v2.2.1) with default parameters. DE analysis was performed on $\mathrm{HRas}^{\mathrm{G} 12 \mathrm{~V}} ; \mathrm{FSOX} 2^{\mathrm{AA} \text { vs DD }}$ and $\mathrm{SOX} 2^{\mathrm{KO}} ; \mathrm{FSOX} 2^{\mathrm{AA} \text { vs DD }}$ conditions. Area proportional diagrams were generated with BioVenn (Hulsen et al., 2008). 
Figure 1. SOX2-phosphorylation reduces clonal expansion in mouse and human SCCs

A

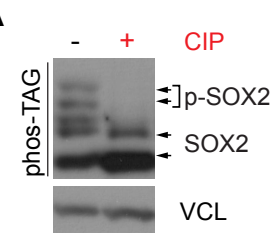

C

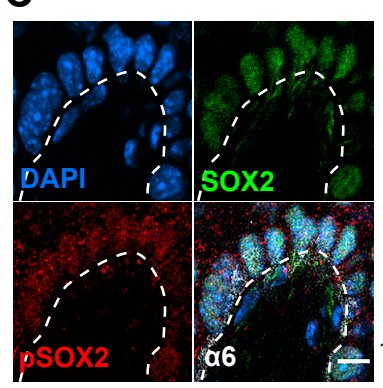

$\mathbf{F}$

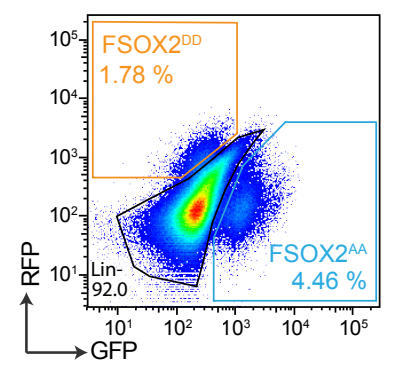

B

D
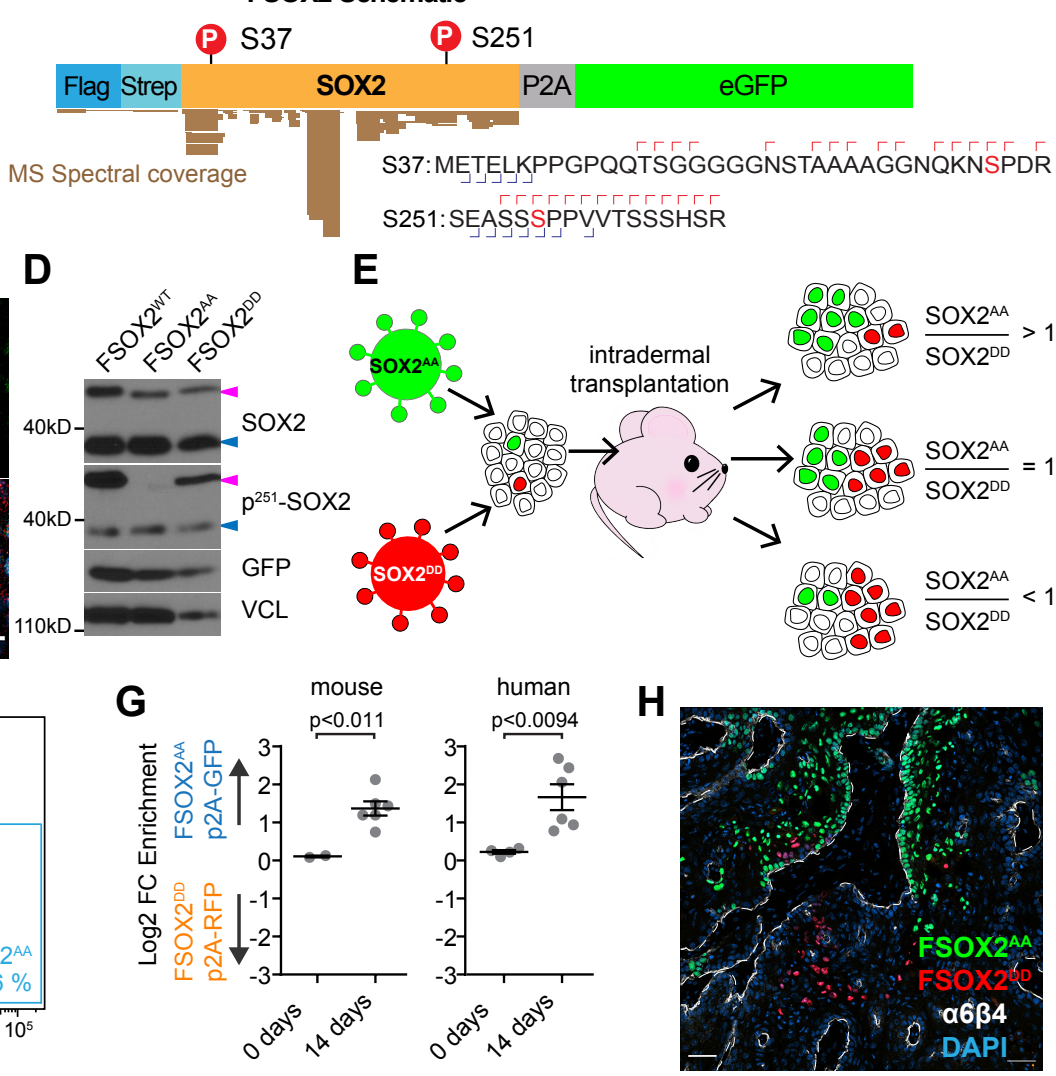

E

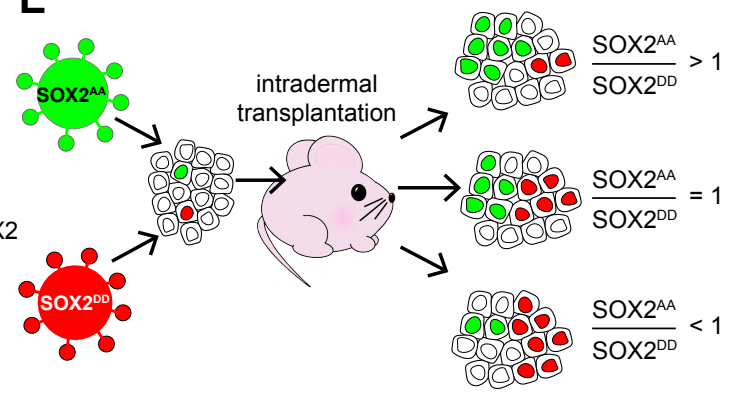

$\mathbf{H}$

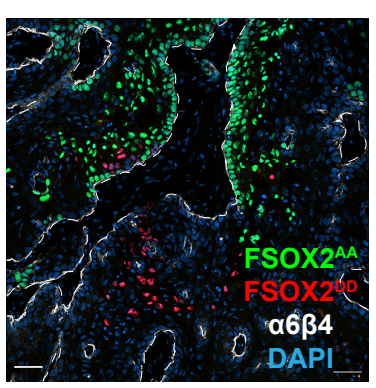


Figure 2. Ectopic SOX2 expression enhances self-renewal and tumor initiation in a $\mathrm{Hras}^{\mathrm{G} 12 \mathrm{~V}}$ driven skin cancer model

A
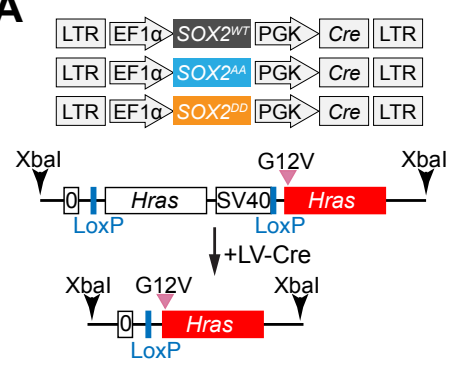

D

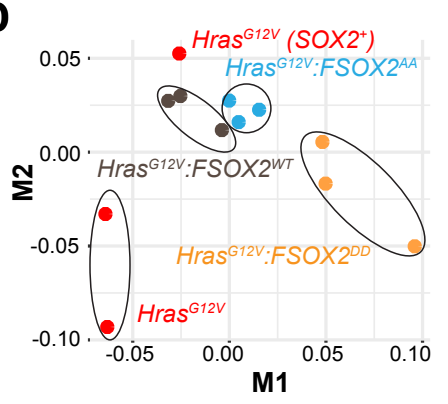

G

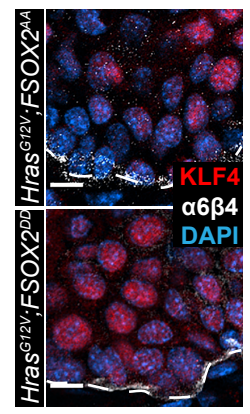

B

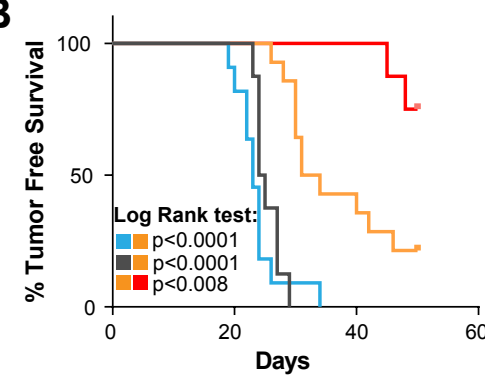

E

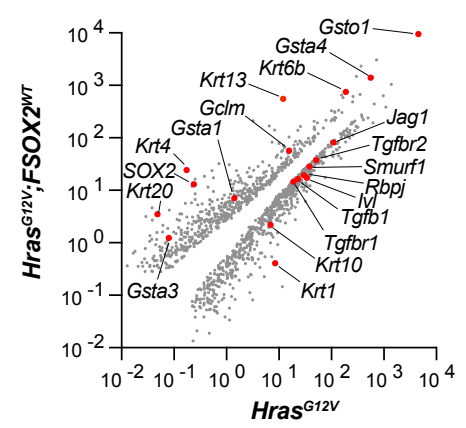

H

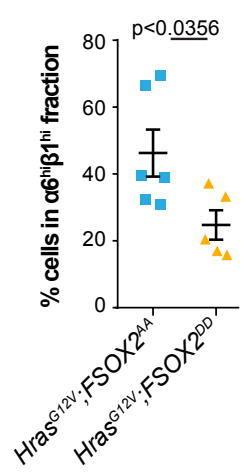

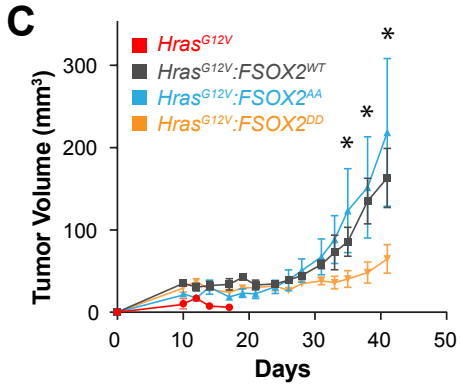

$\mathbf{F}$

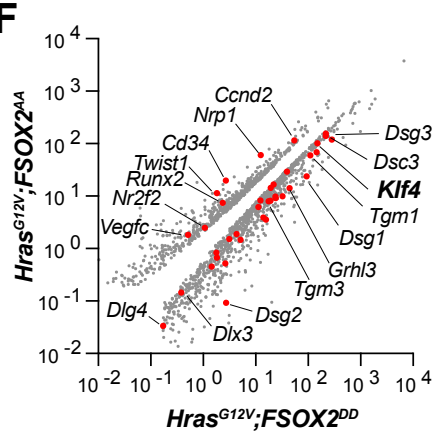

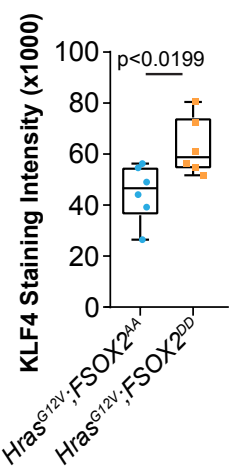
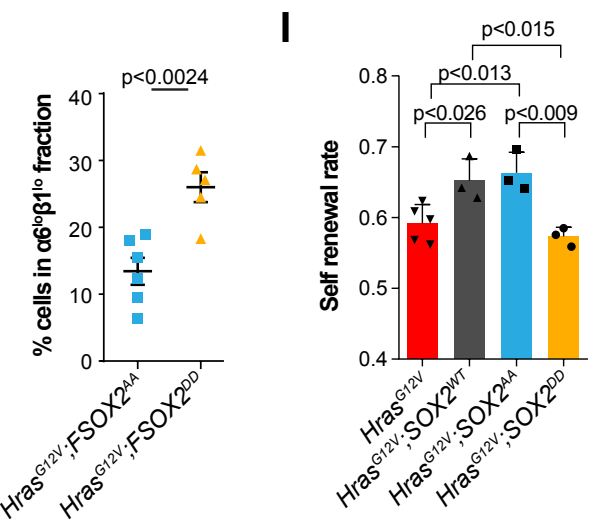


\section{Figure 3. SOX2 phosphorylation enhances differentiation in SCCs}

A
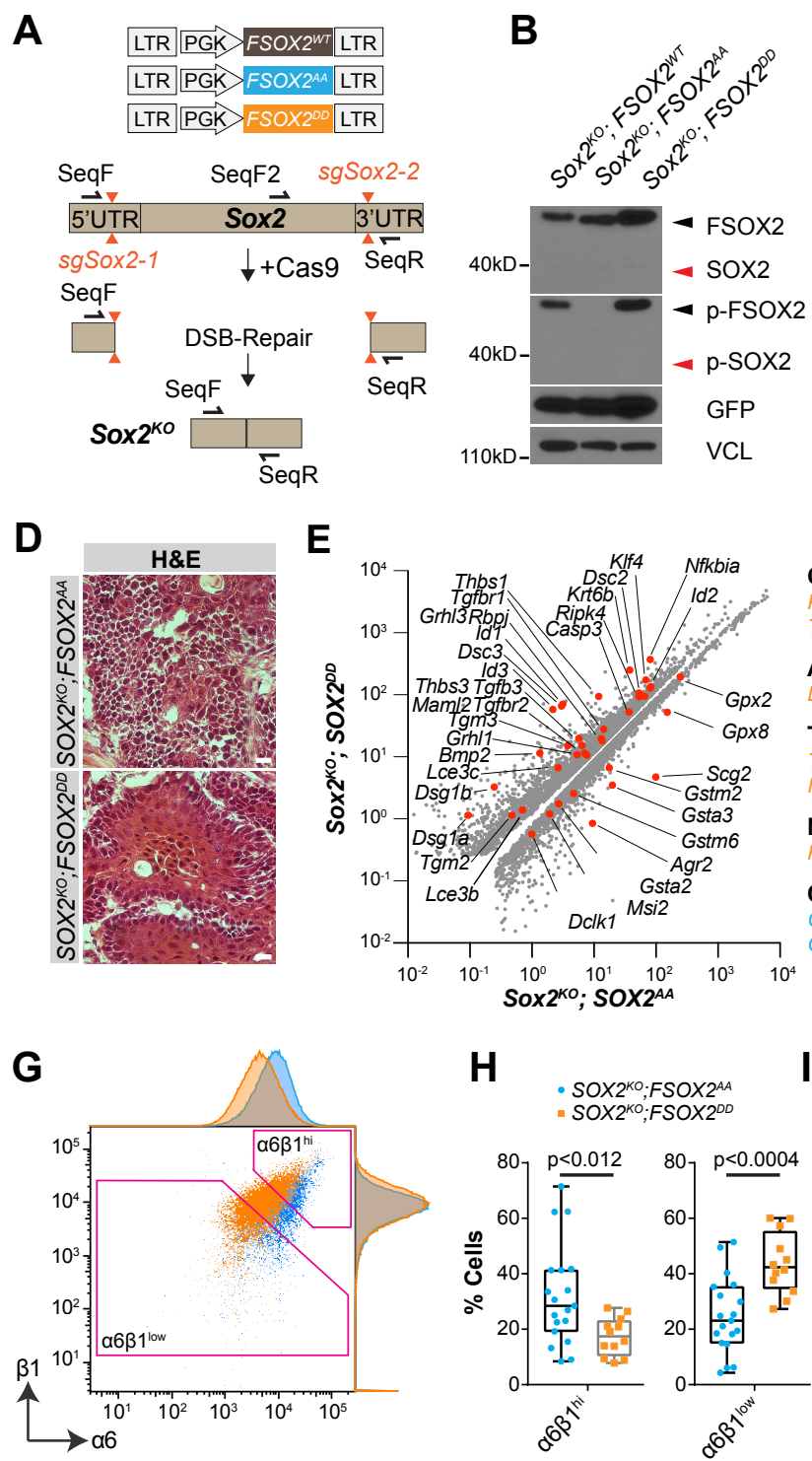

H

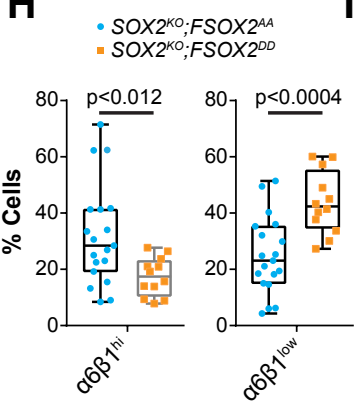

C

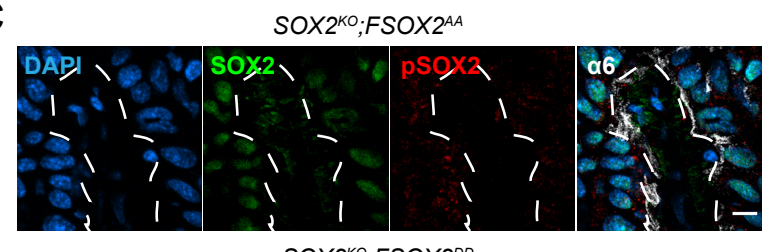

SOX $2^{\mathrm{KO}} ; \mathrm{FSOX} 2^{\mathrm{DD}}$

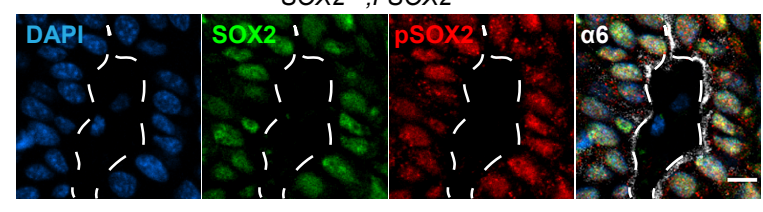

$\mathbf{F}$

Cornification:

KIf4, Grhl1, Grhl3, Tgm1, Tgm2 Tgm3, Lce 3b, Lce3c, Ripk4

Adhesion:

Dsg1a, Dsg1b, Dsg3, Dsc2, Dsc3

TGF $\beta$ :

Tgfbr1, Tgfbr2, Tgfb3, Bmp2, Id1

d2, Id3, Thbs1, Thbs3

Notch:

Rbpj, Mam/2, Hes1, Dll1

Oxidation/Reduction:

Gsta2, Gsta3, Gstm2, Gstm6,
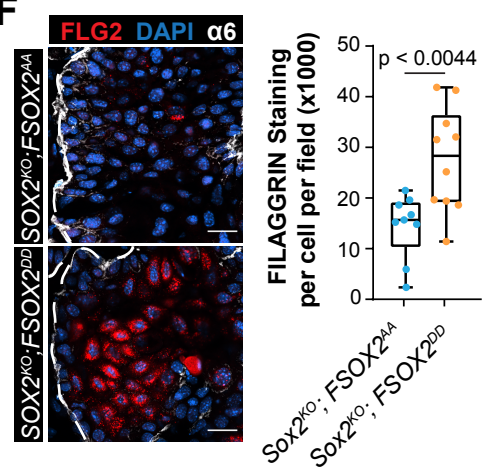

J
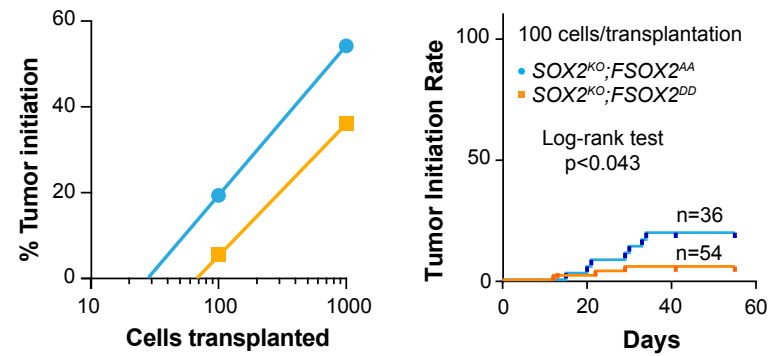


\section{Figure 4. SOX2-phosphorylation affects KIf4 in a small subset of SOX2-bound sites}
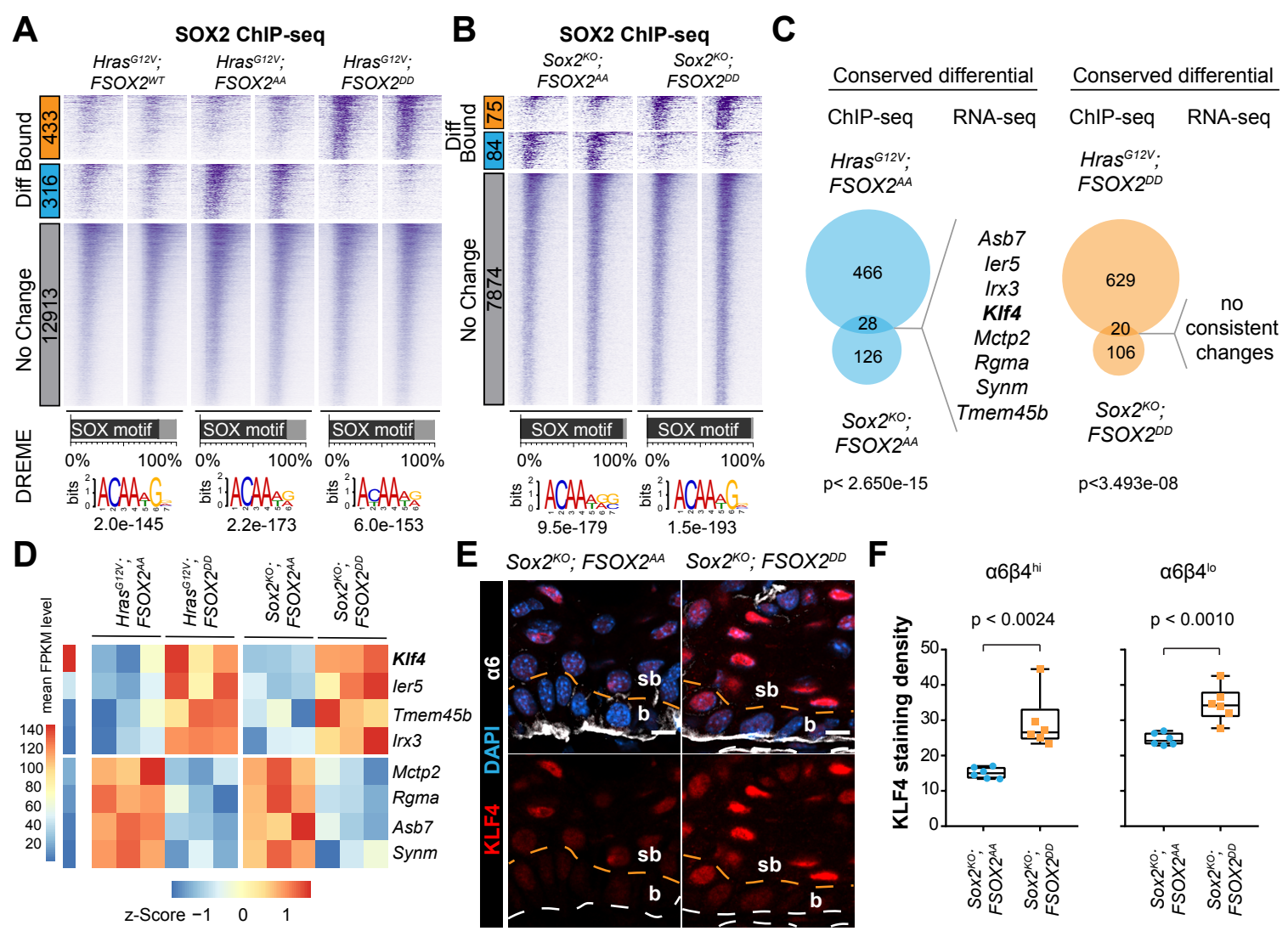


\section{Figure 5. Phosporylated SOX2 is evicted from a KIf4 enhancer to restore KIf4 expression}

A

A

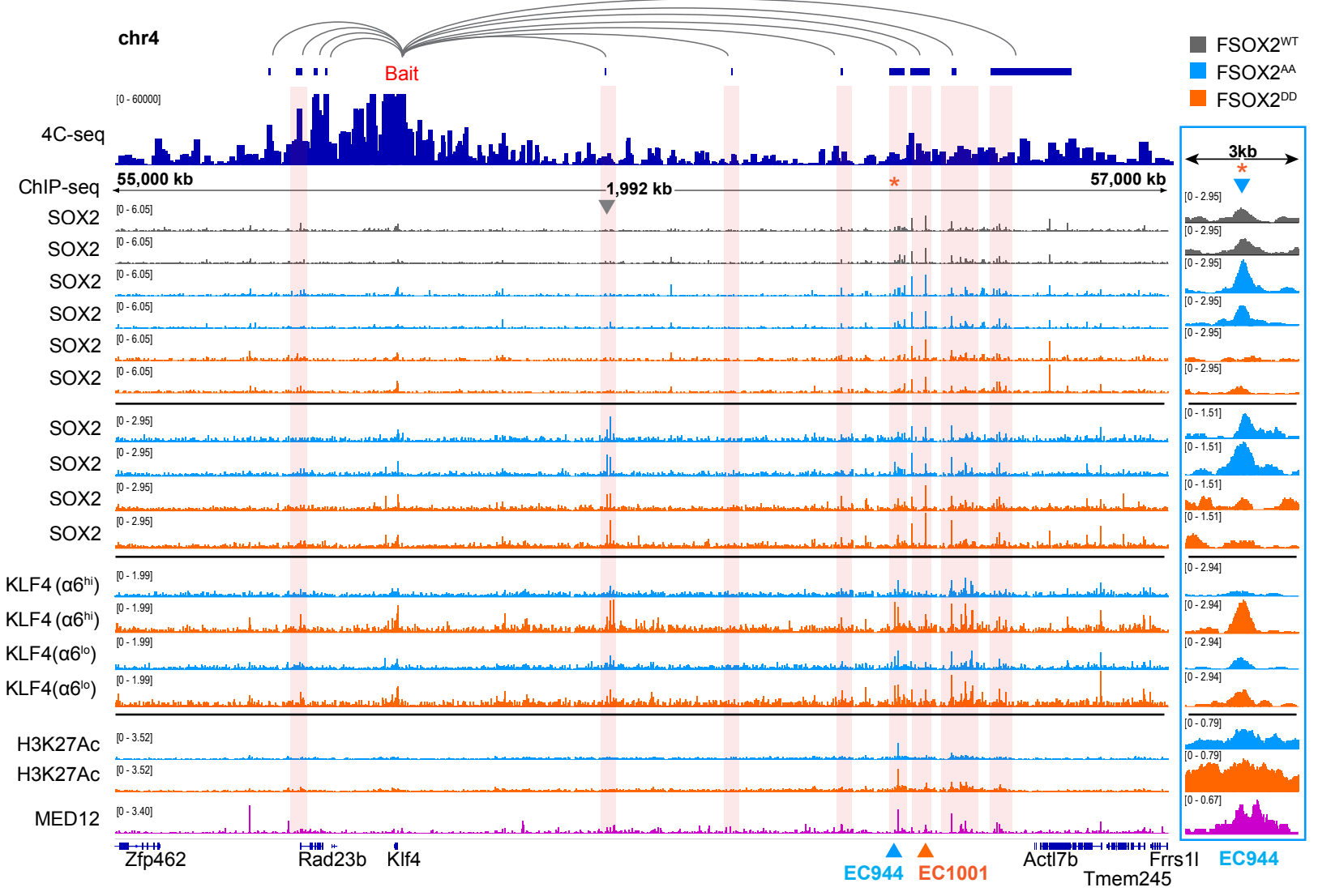

B

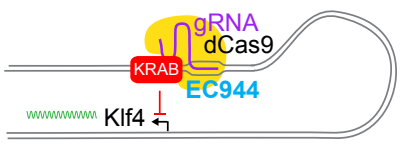

C

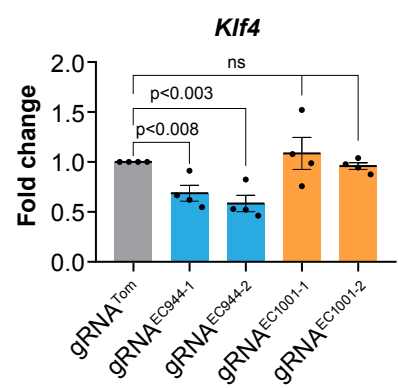

$\mathbf{F}$

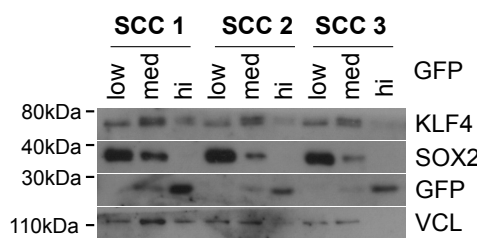

D

EF1a H2BRFP- GFP SV40-EC944

$\mathbf{E}$
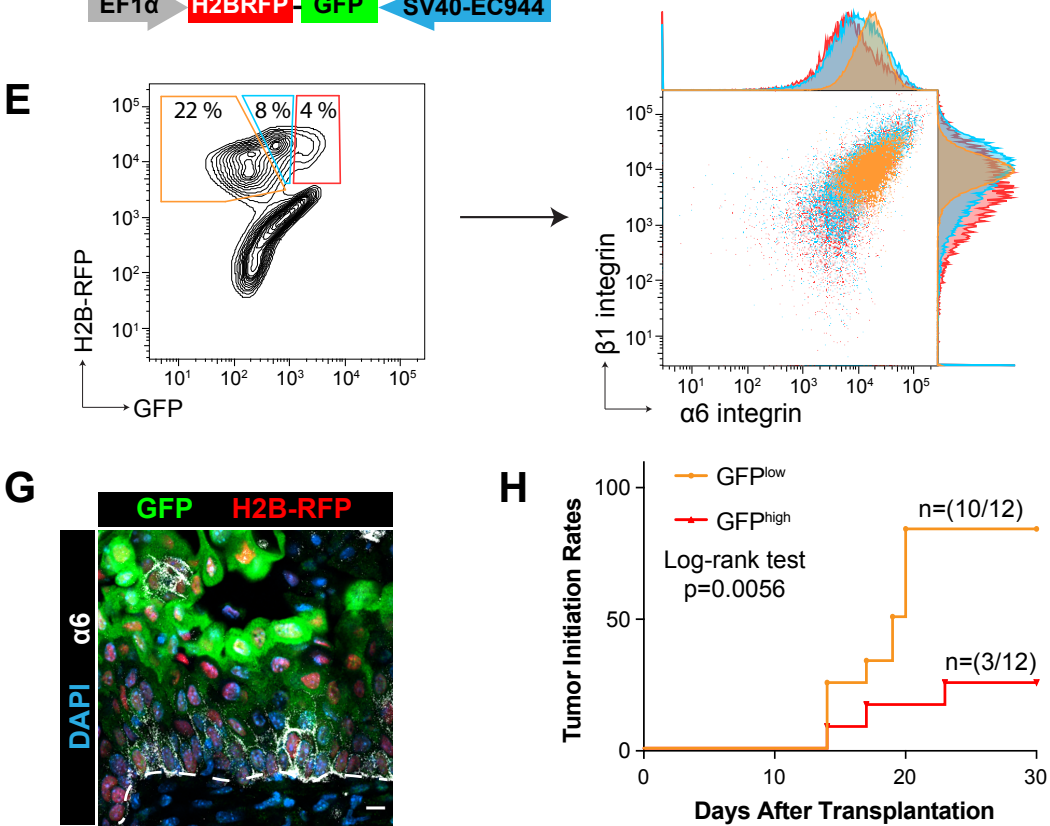
Figure 6. Increased KLF4 expression drives differentiation in F-SOX2 ${ }^{\mathrm{DD}}$ SCCs

A
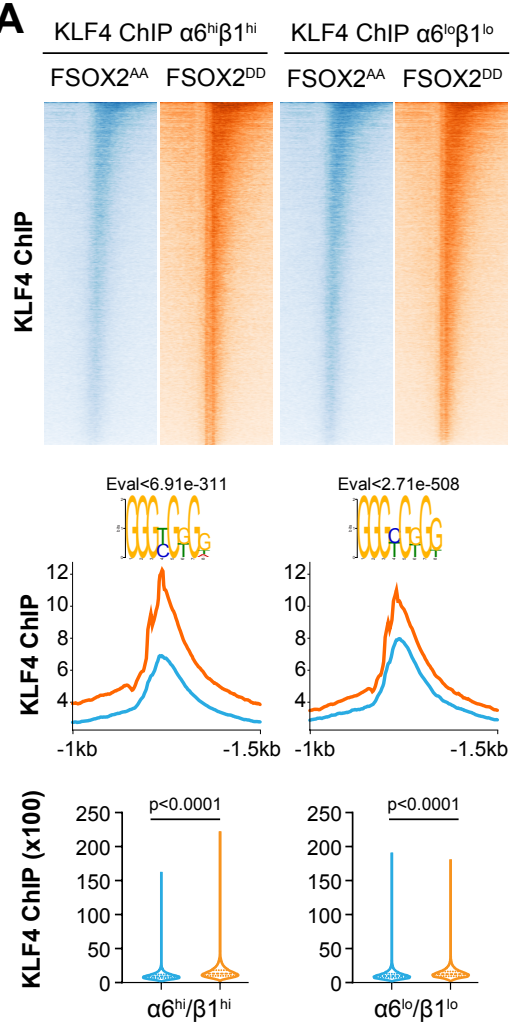

$\mathbf{F}$

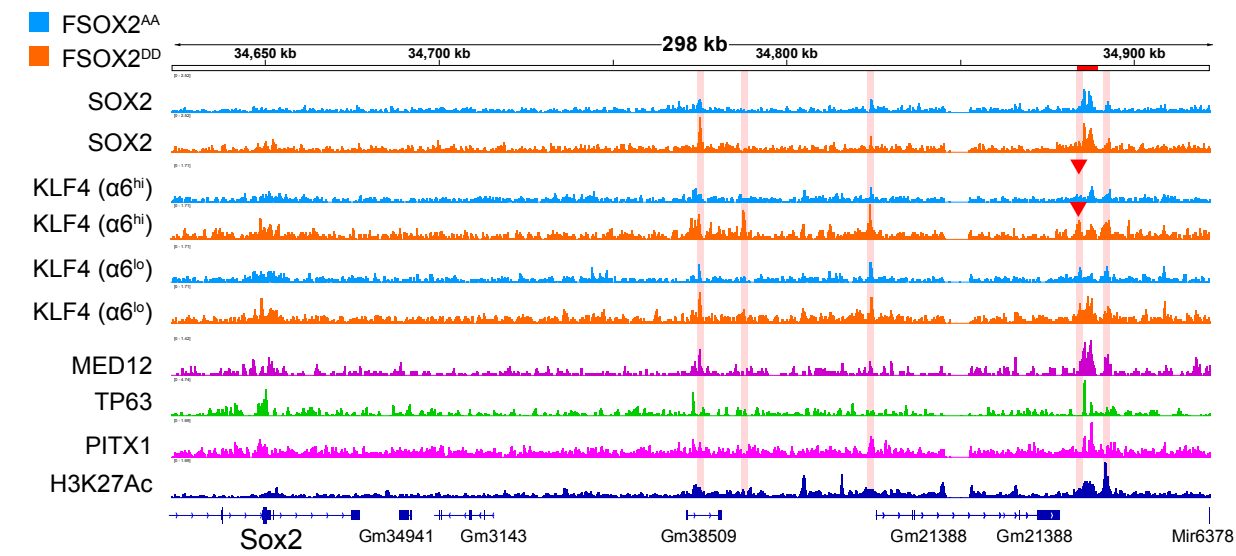

B
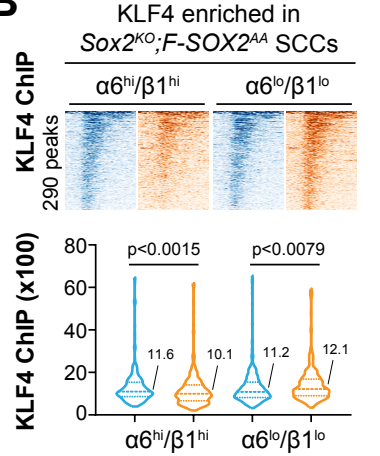

C
E
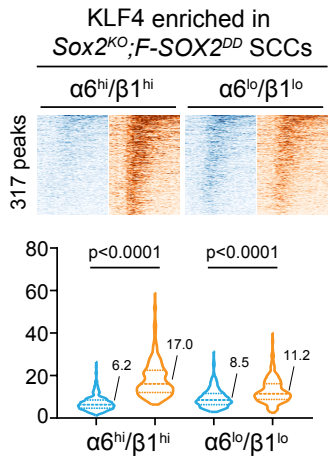

Genes with differential KLF4 enrichment between Sox $2^{K O} ; F-S O X 2^{A A}$ and Sox $2^{K O} ; F-S O X 2^{D D}$
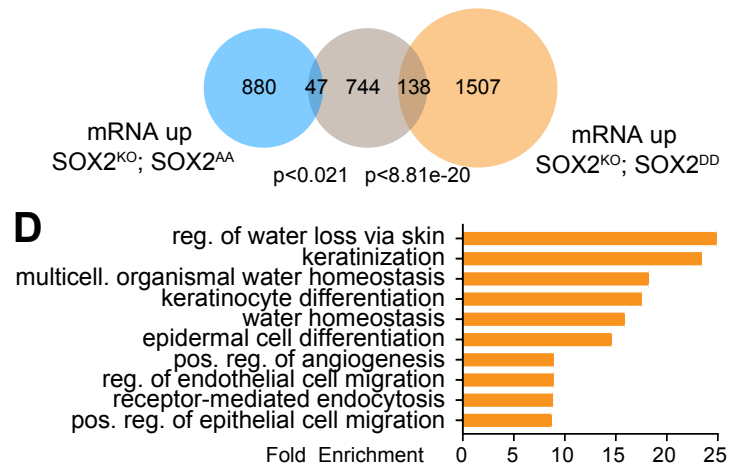

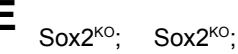

F-SOX2 $2^{A A}$ F-SOX2 ${ }^{D O}$ Gene GO terms

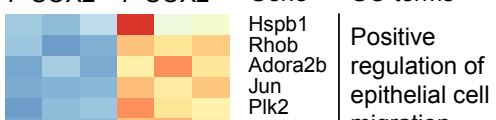

Zc3h12a migration

Tgfbr2

Ptgs2

Emp2

Itgb1

\begin{tabular}{l|l} 
Itgb2 & Receptor \\
Snx9 &
\end{tabular}

Cxcl16 mediated

\begin{tabular}{l|l} 
Cttn & endocytosis \\
Adrb2
\end{tabular}

Klf4

Ubn1

Dll1

Ovol1 Epithelial

Slc9a3r1 Epithelial cell

Epha2 differentiation

Aqp7

Copl

Pyl 26 1 Keratinization

$\underset{\substack{\text { Tmem716 } \\ \text { Krt16 }}}{\text { Reg. of water }}$

\begin{tabular}{l|l} 
Krt16 & Reg. Of water \\
Ugcg
\end{tabular}

Ugcg
Gba

loss via skin
G

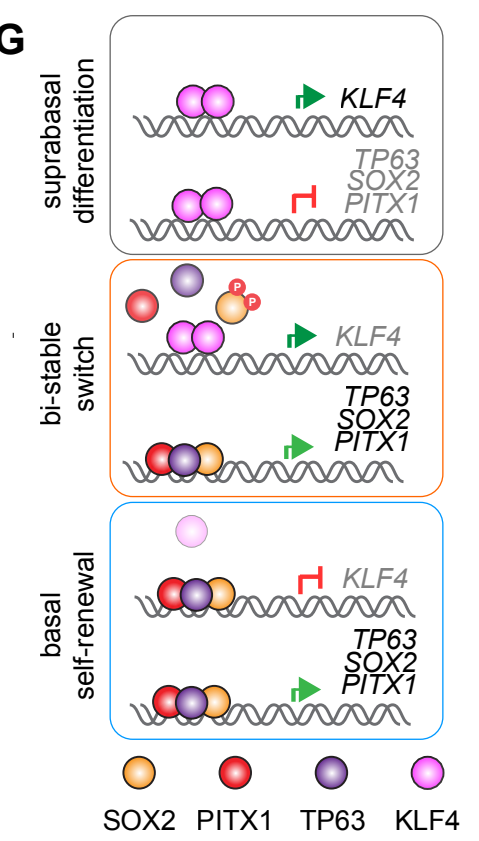

